\title{
Numerical simulation of the performance of a centrifugal pump with a semi- open impeller under normal and cavitating conditions
}

\author{
Georgios Mousmoulis ${ }^{1, *}$, Ioannis Kassanos ${ }^{1}$, George Aggidis ${ }^{2}$, Ioannis Anagnostopoulos ${ }^{1}$ \\ ${ }^{1}$ Laboratory of Hydraulic Turbomachines, School of Mechanical Engineering, National Technical \\ University of Athens, Heroon Polytechniou 9, Zografou, Athens, 15780, Greece \\ ${ }^{2}$ Lancaster University Renewable Energy Group, Department of Engineering, Lancaster \\ University, Bailrigg, Lancaster, LA1 4YW, United Kingdom, \\ ${ }^{*}$ Corresponding author. E-mail address: mousmoulisgeorge@mail.ntua.gr
}

\begin{abstract}
An important flow mechanism that can affect the performance and efficiency, as also the maintenance cost of centrifugal pumps is cavitation. Scientific research has been focusing on the mechanisms that govern cavitation in order to develop experimental and numerical tools that are able either to detect the phenomenon or to anticipate its appearance. In this study, a computational model is used in order to study the cavitation performance of a radial flow centrifugal pump with a semi open impeller. The numerical model includes and studies the effects of the blade tip clearance and its thickness, and it is validated against corresponding laboratory measurements and visualization data obtained for this pump under normal and cavitating flow conditions. The total head drop variation curves versus cavitation parameter are extracted both numerically and experimentally, and compared for various pump loading conditions with satisfactory agreement. A non-periodic pattern of flow and cavitation bubbles between the impeller blades, which is caused by the inlet pipe bending, is also well reproduced by the model. The numerical results display in detail the complex flow field and the secondary flows developed in the tip clearance area, close to the blades leading edge. The effects of the latter on the onset and development of cavitation at the suction side of the impeller, as well as on the backflow cavitation phenomenon are presented and analyzed. Backflow cavitation is found to affect the suction ability of the pump, especially for moderate cavitation conditions, as far as the pressure differences between the two blade sides remain significant.
\end{abstract}

Keywords: Cavitation; Centrifugal pumps; Semi open impeller; Flow visualization; Tip clearance flow; Backflow cavitation

\section{Introduction}

Cavitation is the hydrodynamic phenomenon that can be developed in fluid flow when the static pressure becomes lower than the vapour pressure of the fluid, $p_{\text {sat }}$. In centrifugal pumps such vapour cavities usually appear close to the suction of the impeller at the leading edge (LE) of the blades, where the flow is accelerated as it enters the rotating impeller. These bubbles travel with the flow downstream in the impeller, where the static pressure increases, and they are re-liquified within a few milliseconds. This very violent procedure generates microjets of high intensity that can harm the internal metal surfaces of the pump causing fast and extended ware. Especially in semi-open impellers, the tip clearance, $t_{t c}$, strongly affects the flow through the impeller and the possibility of cavitation appearance. Furthermore, an additional cavitation mechanism has been observed in shroudless impellers, backflow cavitation [1]. The backflow phenomenon becomes apparent usually at low pump flow rates, when the cavities in the suction side are strengthened due to new bubbles that are formed in high speed leakage areas and recirculate to the suction side of the blade and the pump inlet. 
Cavitation in centrifugal pumps has been studied both experimentally and numerically by many researchers. Detailed description of the various tools used for cavitation laboratory measurements can be found in Mousmoulis et al. study [2]. In computational modelling, various approaches have been proposed in order to study the formation of vapour cavities in the flow around bluff bodies, hydrofoils, and in nozzles and hydraulic turbomachines. The development of such models is a process of increased complexity, since the accurate simulation of cavitation requires the consideration of several parameters, such as i) the variation of density between the two phases, ii) the mass and energy between the two phases, iii) the relative motion (slip) between the phases, and iv) the irregular shape of the interface boundary. Moreover, a model must overcome the problems related to stability and robustness of the numerical procedure, so as to be applicable and efficient. The inability to satisfy all the above requirements by the development of one common numerical approach justifies the existence of a variety of numerical models, which make different assumptions and simplifications. The appropriate selection of the modelling approach depends on the individual characteristics of the problem investigated and the computational resources.

The numerical methodologies that aim to simulate cavitation can be split in two main categories [3, 4]: i) the Interface tracking methods (ITM), and ii) the Interface capturing methods (ICM). The ITM provide the iterative solution of the incompressible liquid flow field, along with the interface boundary of the attached vapour area. The latter is treated as an isosurface in the liquid's computational domain, at which the pressure equals the vapour saturation pressure. The iterations initiate after a first estimation of the attached vapour cavity size, which is progressively updated until a converged shape is achieved. In order to ensure that the vapour interface matches the constant vapour pressure value, a wake closure model is employed for the wake region [5]. This approach has been found capable to predict successfully the extent of the cavity [3, $4 \& 6]$ and the machine's performance deterioration under heavy cavitating conditions [7]. Nevertheless, ITMs face difficulties when they are used in threedimensional flows, since they are not able to accurately track the three-dimensional wake $[8$, 9]. In addition, ITMs are not able to model cavitation types other than attached vapour formations.

In the case of ICMs, the entire flow domain is solved, while the density of the medium varies between the vapour and the liquid density. Unlike the ITMs, ICMs allow for the modelling of all types of vapour formation, included bubble, vortex and tip cavitation. Two different approaches are used towards the calculation of the medium's density: i) the Barotropic Approach (BA) and ii) the Transport Equation-based Models (TEM). In the first case, the density is associated with the local static pressure, with the use of a barotropic state law [10], while both phases are treated as a homogeneous mixture fluid. This approach assumes that there is not mass exchange between the vapour and the liquid phase. Barotropic models have been used successfully by several authors for the simulation of two phase flow development in hydrodynamic geometries [5, 11-18]. In all these cases, the heat transfer between the two phases is ignored and the two phases are assumed to be in a complete thermodynamic equilibrium. It is noted that Coutier-Delgosha et al. [13] proposed the replacement of the density in the calculation of turbulent viscosity with an expression that takes into account the compressibility effects in the medium under cavitating conditions. The new expression has been found to improve the predictions of the actual local flow conditions and the shape of the cavity [13], as well as of the trend of the characteristic curves of the pump under cavitating conditions [19]. This modification has been applied in both barotropic and TEM approaches [20-23].

On the other hand, the TEM incorporate a transport equation for the mass and volume fraction that regulates the mass transfer between the two phases. The transport equation is usually based on a simplified version of the main equation of bubble dynamics, the RayleighPlesset equation, which relates bubble growth with the internal bubble pressure conditions [24]. A great number of models have been proposed in order to describe the net mass transfer between the liquid and vapour phase at the bubble interface. The most frequently used models in the area of hydraulic turbomachinery follow the homogeneous approach (similarly to the barotropic models), which assumes the vapour and liquid phase to behave as one medium. The models are briefly presented in Table 1 and as can be seen, all expressions are functions of the 
main quantities and properties of the fluid (e.g., flow, vapour, mixture and liquid densities, $\rho$, $\rho_{v}, \rho_{m}$ and $\rho_{l}$, vapour and non-condensable gas volume $\alpha_{v}, \alpha_{n c g}$ and mass fractions, $f_{v}, \mathrm{f}_{\mathrm{ncg}}$, static pressure, $p$, turbulence kinetic energy, $k$, free stream velocity, $u_{\infty}$ and saturation pressure, $\left.p_{\text {sat }}\right)$, and usually contain one or more empirical coefficients (e.g. bubble radius, $R_{B}$, nucleation site volume fraction, $\alpha_{n u c}$, and vaporization and condensation factors, $C_{v a p}$ and $\left.C_{c o n d}\right)$. Some of the expressions developed $[25,26]$ allow also the modelling of non-condensable gas in the mixturemedium $\left(\alpha_{\text {ncg }}\right.$, and $\left.f_{n c g}\right)$, in order to include their effect on the development of vapour phase, which is found to be significant especially during cavitation inception conditions, in some experimental and numerical studies [27-32]. Li [33] showed that a mixture with noncondensable gas content higher than $15 \mathrm{ppm}$ can even change significantly the critical cavitation number value for pump head collapse.

The Kunz et al. [25] model that is described in Eq. 1, uses an expression that relates evaporation $\left(\mathrm{m}^{-}\right)$with the liquid volume fraction, $\alpha_{l}$, and the difference between static pressure, $p$, and saturation pressure, $p_{\text {sat }}$. This model is used in $[34,35]$ for the study of sheet cavitation in a centrifugal pump for a wide range of flow rates, $\Phi$ and cavitation numbers, $\sigma$. Medvitz et al. [34] conducts steady state simulations for flow rates close to the best efficiency point (BEP) and transient simulation for partial loading, where additional unsteadiness is introduced to the flow in the impeller. Wang et al. [35] introduced the effect of turbulent pressure fluctuations to the Kunz et al. model, as it is proposed by Singhal [26]. The new model is compared with the original Kunz et al. and Zwart et al. models [36], and its results show satisfactory agreement regarding the cavitation curves of the machine but also the prediction of the total head breakdown and the shape of the cavity formed at the pump suction The Schnerr and Sauer model [37] uses the Rayleigh-Plesset equation to derive the expressions of Eq. 2 that contain no empirical constants. Sato et al. [38] use Schnerr and Sauer model in order to predict two phase flow phenomenon in a double suction volute pump by Large Eddy Simulation and the k$\varepsilon$ turbulence model. Their results showed that the LES can predict more accurately the detailed characteristics of the cavities and the pressure waves derived by bubble implosion, especially in cases where cavities are followed by flow vortices.

The full cavitation model (FCM) presented by Singhal et al. [26] in 2002 is based on the Rayleigh-Plesset equation and its novelty is related with the introduction of the turbulent kinetic energy, $\mathrm{k}$, and the bubble surface tension, $\mathrm{s}$, in the mass transfer equation (3) (Table 1). Mostafa et al. [39] validate the numerical results derived with FCM with experiments made for the study of the tip cavitation in an axial flow pump. Ding et al. [40] develop an innovative meshing tool able to generate unstructured body-fitted Cartesian grid, similarly to Anagnostopoulos [41]. This tool is applied with the FCM in an axial centrifugal pump in order to use it both as design and diagnostic tool. The main advantages of this method is the fast mesh generation in complex geometries, while the results are similar with denser structured meshes. Kim et al. [42] manage to improve the accuracy of the onset and development of cavities through transient simulation and analysis in a radial pump with time step that corresponded to $3^{\circ}$ rotation.

Finally, the Zwart et al. model [36] is based in the expression developed by Iben [43] and relate evaporation and condensation source terms of Eq. 4 (Table 1) with the bubble radius and its dynamics. Pierrat et al. [44] use this model and simulate a helicon-centrifugal pump in order to assess two different meshing methodologies; a blade to blade topology (BTBT) and one that included the entire pump geometry. Their results show that BTBT calculations can provide acceptable results, and that the FCM model can predict accurately the size and position of the bubbles. Liu et al. [45] use Zwart et al. model for the investigation of cavitation phenomenon in a pump-turbine and confirm that bubble formation at the inlet of the pump could heavily reduce the efficiency and the performance of the machine. Cunha and Nova [46] model the cavitation performance in a radial impeller by studying only the BTBT. The model is found capable to predict the two phase flow, since all cavitation pocket areas appear at the expected positions, at the suction of the impeller close to the blades inlet. Zhang et al. [22, 47-48] investigates the structure of cavitation patterns in tip leakage vortex cavitation of an axial pump found that the tip leakage vortex trajectory is heavily influenced by the flow rate and blade loading. The Zwart et al. model has also been used successfully by the present authors for the study of cavitating draft tube vortex in a Francis turbine $[49,50]$. 
Table 1. Equations of cavitation models used in centrifugal pumps numerical simulations.

Kunz et al. [25]

$$
\dot{m}^{-}=\frac{C_{\text {vap }} \rho_{v} \alpha_{l}}{0.5 \rho_{l} u_{\infty}^{2} t_{\infty}}\left(p-p_{\text {sat }}\right) \quad \dot{m}^{+}=\frac{C_{c o n d} \rho_{v}\left(\alpha_{l}-\alpha_{n c g}\right)^{2}}{t_{\infty}}\left(1-\alpha_{l}-\alpha_{n c g}\right)
$$

\section{Schnerr and Sauer [37]}

$$
\dot{m}^{-}=-\frac{\rho_{v} \rho_{l}}{\rho_{m}} \alpha_{v}\left(1-\alpha_{v}\right) \frac{3}{R_{B}} \sqrt{\frac{2\left(p_{s a t}-p\right)}{3 \rho_{l}}} \quad \dot{m}^{+}=\frac{\rho_{\nu} \rho_{l}}{\rho_{m}} \alpha_{v}\left(1-\alpha_{v}\right) \frac{3}{R_{B}} \sqrt{\frac{2\left(p-p_{s a t}\right)}{3 \rho_{l}}}
$$

\section{Full Cavitation Model [26]}

$$
\dot{m}^{-}=-C_{\text {vap }} \frac{\sqrt{k}}{s} \rho_{\nu} \rho_{l} \sqrt{\frac{2\left(p_{\text {sat }}-p\right)}{3 \rho_{l}}}\left(1-f_{v}-f_{n c g}\right) \quad \dot{m}^{+}=C_{\text {cond }} \frac{\sqrt{k}}{s} \rho_{l} \rho_{l} \sqrt{\frac{2\left(p-p_{\text {sal }}\right)}{3 \rho_{l}}} f_{v}
$$

\section{Zwart et al.[36]}

$$
\dot{m}^{-}=-C_{v a p} \frac{3 \alpha_{n u c}\left(1-a_{v}\right) \rho_{v}}{R_{B}} \sqrt{\frac{2\left(p_{\text {sat }}-p\right)}{3 \rho_{l}}} \quad \dot{m}^{+}=C_{\text {cond }} \frac{3 \alpha_{v} \rho_{v}}{R_{B}} \sqrt{\frac{2\left(p-p_{\text {sat }}\right)}{3 \rho_{l}}}
$$

In the present study, the Zwart et al. model [36] is used together with commercial CFD software for the study of cavitation development in a centrifugal pump with a radial semi-open impeller, which, to our knowledge, has not reported in previous scientific articles. The majority of relevant published works deal with the effect of tip clearance in axial [22, 47-48 \& 51-55], mixed flow pumps [56-62], and inducers [63-65]. This work examines also the effect of tip clearance and its geometrical characteristics on the suction and backflow cavitation formation for a wide range of pump flow rate and cavitation intensity. The numerical results are validated against corresponding laboratory measurements taken for this particular pump type, while the exact inlet and outlet pipe configuration of the test rig is also simulated. More specifically, the validation of the pump performance curves is followed by a detailed comparison of the developed vapour zone, from the cavitation inception point until total head breakdown.

\section{Numerical modelling}

\subsection{Mathematical formulation}

The Zwart et al. model [36] is a homogeneous model in which vapour and liquid phases are considered to behave as one medium (mixture) rather than two separate phases that interact with each other. Hence, only one set of governing equations is solved, and it is assumed that the two phases have the same dynamics and there is no slip between them. The governing flow equations thus become:

$$
\begin{aligned}
& \frac{\partial \rho_{m}}{\partial t}+\frac{\partial}{\partial x_{j}}\left(\rho_{m} u_{j}\right)=0 \\
& \frac{\partial}{\partial t}\left(\rho_{m} u_{j}\right)+\frac{\partial}{\partial x_{j}}\left(\rho_{m} u_{i} u_{j}-\tau_{i j}\right)=-\frac{\partial p}{\partial x_{i}}+\rho_{m} g+F
\end{aligned}
$$

where $p$ represents the pressure flow field, $F$ the external body forces and $u_{j}$ the flow field velocity and $\tau_{i j}$ the viscous stress tensor. The density of the mixture, $\rho_{m}$ and the vapour transfer equation that controls the mass transfer between the two phases are given by the following corresponding expressions: 
$\rho_{m}=\rho_{v} \alpha_{v}+\rho_{l}\left(1-\alpha_{v}\right)$

$\frac{\partial}{\partial t}\left(a_{l} \rho_{l}\right)+\frac{\partial}{\partial x_{j}}\left(a_{l} \rho_{l} u_{j}\right)=\dot{m}^{-}+\dot{m}^{+}$

where $\alpha_{v}$ and $a_{l}$ are the vapour and liquid volume fraction, respectively. The equations that describe the mass transfer evaporation, $\dot{m}^{-}$and condensation, $\dot{m}^{+}$are given by Eqs. 4 in Table 1. The values for the model coefficients used in this study are: $R_{B}=10^{-6} \mathrm{~m}, \alpha_{\text {nuc }}=0.0005, C_{\text {vap }}=50$ and $C_{\text {cond }}=0.01$. These values are proposed by Zwart et al. (2004) and have been found to work decently in studies related with two phase flow modelling in semi-open radial hydraulic turbomachinery applications [45, 47-48 \& 66-67].

In the present study, the k- $\omega$ SST turbulence model [68] is used for the closure of Reynoldsaveraged Navier-Stokes solver for a more accurate prediction of flow separation under cavitating and part load conditions.

$$
\begin{aligned}
& \frac{\partial}{\partial t}\left(\rho_{m} k\right)+\frac{\partial}{\partial x_{j}}\left(\rho_{m} k u_{j}\right)=\frac{\partial}{\partial x_{j}}\left(\Gamma_{k} \frac{\partial k}{\partial x_{j}}\right)+G_{k}-Y_{k} \\
& \frac{\partial}{\partial t}\left(\rho_{m} \omega\right)+\frac{\partial}{\partial x_{j}}\left(\rho_{m} \omega u_{j}\right)=\frac{\partial}{\partial x_{j}}\left(\Gamma_{\omega} \frac{\partial k}{\partial x_{j}}\right)+G_{\omega}-Y_{\omega}+D_{\omega}
\end{aligned}
$$

In the above equations, $\omega$ represents the specific dissipation rate, $D_{\omega}$ is the cross-diffusion term, while $\Gamma_{k}$ and $\Gamma_{\omega}, G_{k}$ and $G_{\omega}$ and $Y_{k}$ and $Y_{\omega}$ represent the effective diffusivity, the generation and the dissipation of the turbulence variables $k$ and $\omega$, respectively.

\subsection{Performance equations}

The following Eqs. 11 and 12 are used for the calculation of the total head, $H_{t o t}$, and efficiency, $\eta$ of the pump, respectively:

$$
\begin{aligned}
& H_{\text {tot }}=H_{\text {tot_Dis }}-H_{\text {tot_Suc }}=\left(\frac{p_{\text {Dis }}-p_{\text {Suc }}}{\rho g}\right)+\left(\frac{v_{\text {Dis }}^{2}-v_{\text {Suc }}^{2}}{2 g}\right)+Z \\
& \eta=\frac{\rho g H_{\text {tot }} Q}{P_{\text {mech }}}=\frac{\rho g H_{\text {tot }} Q}{P_{\text {el }} \eta_{\text {mot }}}
\end{aligned}
$$

where $v_{D i s} v_{S u c}, p_{D i s}$ and $p_{S u c}$ are the mean pipe velocity and static pressure at discharge and suction, respectively, $\mathrm{Z}$ is the vertical distance between the discharge and suction measuring points (see section 3), $Q$ the flow rate, $P_{\text {mech }}$, is the mechanical power, $P_{e l}$, is the electric power consumed from the motor and $\eta_{m o t}$ the motor's efficiency. For the analysis of the two phase flow inside the pump the dimensionless Thoma's cavitation number, $\sigma$, is used, given by Eq. 13. The Net Positive Suction Head (NPSH) depicts the difference between the total pressure in the suction side, $H_{\text {tot_Suc }}$ from the vapour pressure, $H_{V}$, both expressed in head units. Finally, the dimensionless parameters of the pump for the flow rate, $\Phi$, head, $\Psi$, and power, $N$, are introduced by Eqs. 14, 15 and 16 respectively:

$$
\begin{aligned}
\sigma & =\frac{N P S H}{H_{\text {tot }}}=\frac{H_{\text {tot_Suc }}-H_{V}}{H_{\text {tot }}} \\
\Phi & =\frac{c_{2 n}}{u_{2}} \\
\Psi & =\frac{2 g H_{\text {tot }}}{u_{2}^{2}} \\
N & =\frac{P_{\text {mech }}}{\rho \Omega^{3} D^{5}}
\end{aligned}
$$


where $c_{2 n}$ is the radial component of the absolute flow velocity at the impeller outlet, $u_{2}$ the circumferential velocity of the impeller and $D$ the impeller diameter.

\subsection{Numerical grid and computational details}

The computational domain consists of four zones (Fig. 1a): The inlet pipe (I), the impeller nozzle (II), the impeller blades (III) and the volute with the outlet pipe (IV). The semi open impeller with diameter, $D=2 R_{2}=165 \mathrm{~mm}$, shown in Fig. 1b, consists of twelve radial blades $\left(\beta_{1}=\beta_{2} \sim 90^{\circ}\right)$. The domain includes the original tip clearance between the blades and the casing, with thickness $t_{t c}=0.9 \mathrm{~mm}$. The impeller geometrical characteristics are provided in Table 2 . The specific speed value of Table 2 is calculated by Eq. 17, while the units used for the flowrate, the total head and rotational speed are $\mathrm{m}^{3} \mathrm{hr}^{-1}$, meters and rpm, respectively:

$$
n_{q}=\Omega \frac{Q^{0.5}}{H_{t o t}^{0.75}}
$$
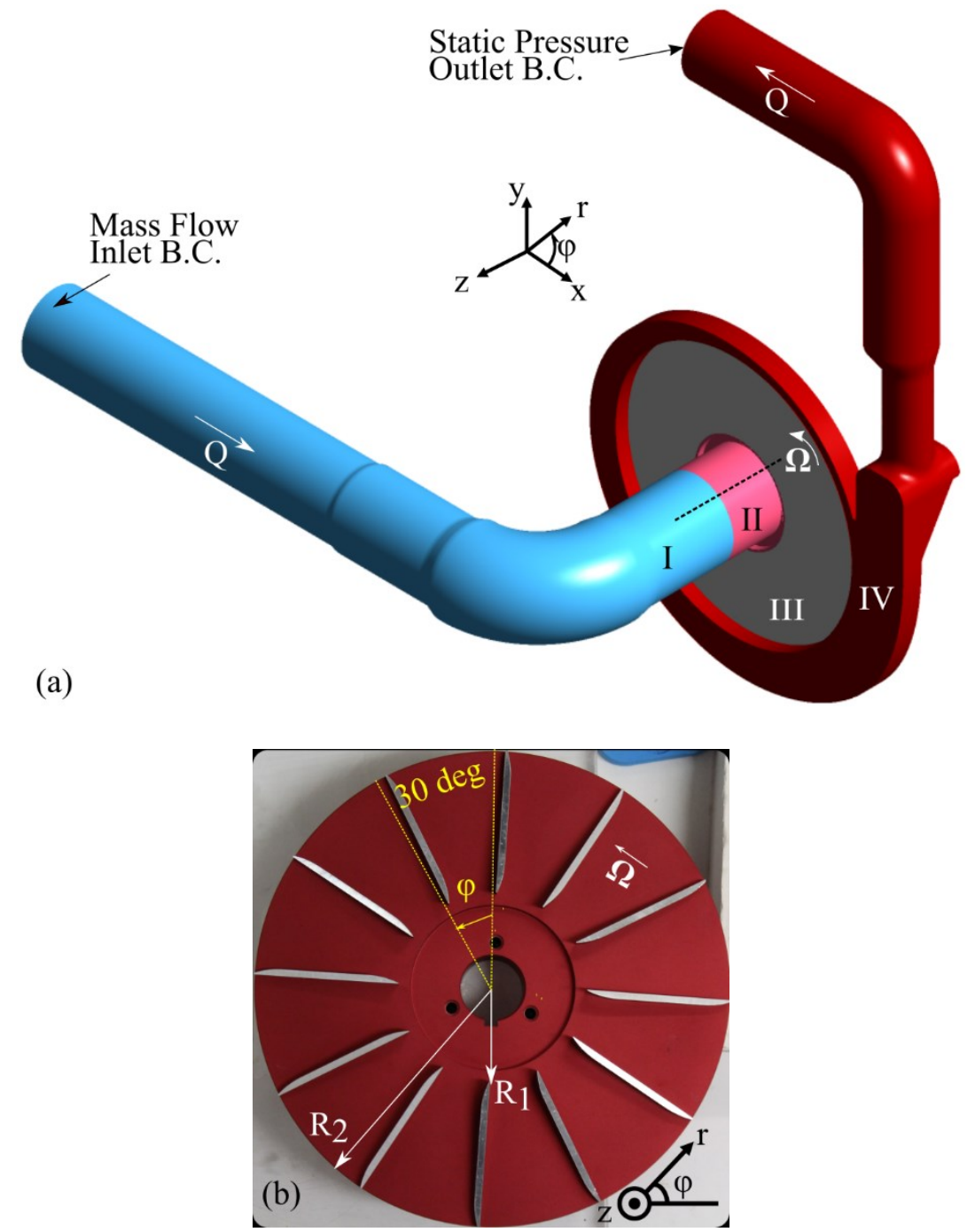

Fig. 1. (a) The computational domain, (b) the simulated impeller.

Table 2 Impeller Geometrical Characteristics.

\begin{tabular}{ccccccc}
$\begin{array}{c}\text { Specific speed } \\
\mathbf{n}_{\mathbf{q}}[\mathrm{rpm}]\end{array}$ & $\begin{array}{c}\text { No of } \\
\text { Blades }\end{array}$ & $\mathbf{b}_{1}$ & $\mathbf{t}_{\mathrm{tc}}$ & $\mathbf{R}_{1}$ & $\mathbf{R}_{2}$ & $\mathbf{\Omega}$ \\
{$[\mathrm{mm}]$} & {$[\mathrm{mm}]$} & {$[\mathrm{m}]$} & {$[\mathrm{m}]$} & {$[\mathrm{rpm}]$} \\
\hline
\end{tabular}




\begin{tabular}{lllllll}
738 & 12 & 7.53 & 0.9 & 0.033 & 0.083 & 1800 \\
\hline
\end{tabular}

The rotating zone II of the impeller is meshed with tetrahedral elements, whereas the blades zone III is meshed as BTBT in ANSYS Turbogrid with hexahedral elements. In this study, the modelling of the single and two-phase flow conditions at the tip clearance of the impeller is of crucial importance, so ten hexahedral elements are used for the spatial discretisation at the zdirection (Fig. 2) of the gap, as a good compromise of local discretization accuracy and quality of the mesh. The zones I and IV are meshed with tetrahedral elements, except of the straight pipes, where the discretization was done with hexahedral elements. The meridional view from the final mesh of the total domain is presented in Fig. 2 that includes the four zones with the corresponding interfaces.

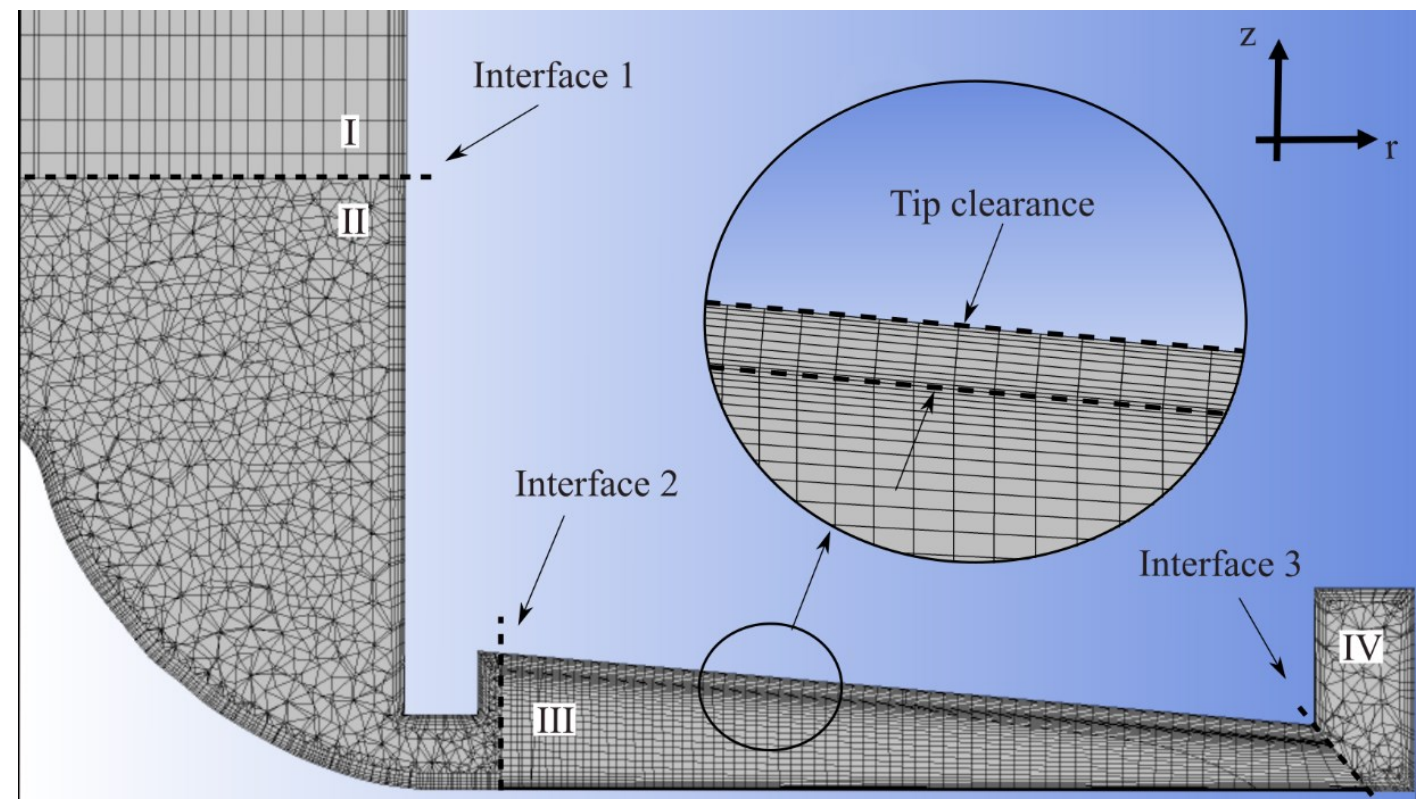

Fig. 2 Meridional view of the computational domain of mesh No. 2.

The mesh convergence study involved the flow simulation at $\Phi=0.074$ and $\sigma=0.33$, using four meshes of different density in order to study the spatial convergence at cavitating conditions. The results are presented in Fig. 3a. The coarse mesh (Mesh No. 1) had 1.8 million elements and was refined five times by keeping the average $\mathrm{y}^{+}$value below 10 . The structure of meshes No. 1, $2 \& 4$ at the impeller inlet area, which is the crucial region in terms of cavitation development, are given in Figs. 3b, 3c \& 3d. Fig. 3a shows that that all parameters do not change significantly for meshes No. 2, 3 and 4.

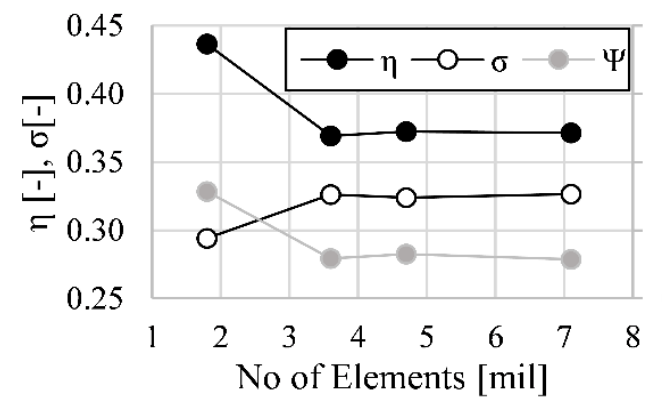

(a)
1.075

1.050

$1.025 \frac{7}{3}$

1.000

0.975

(a)

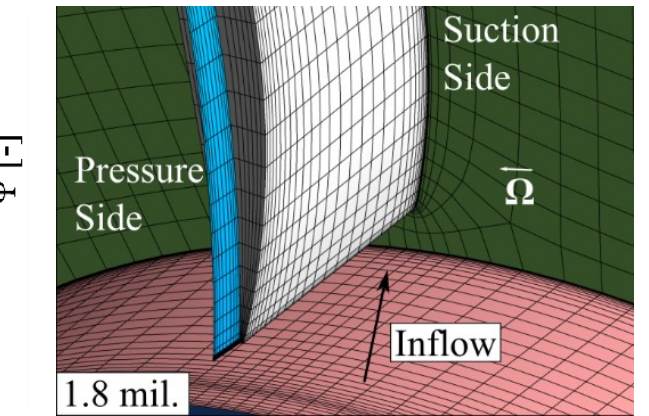

(b) 


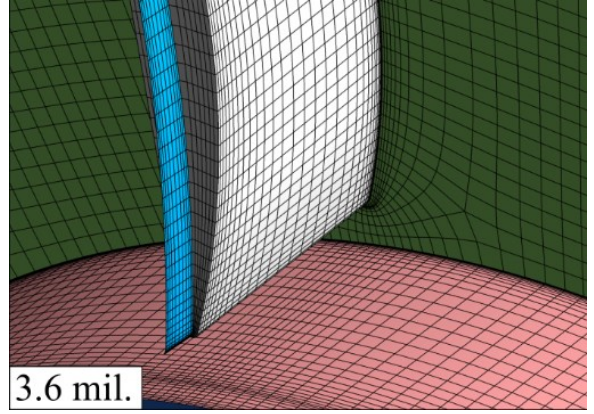

(c)

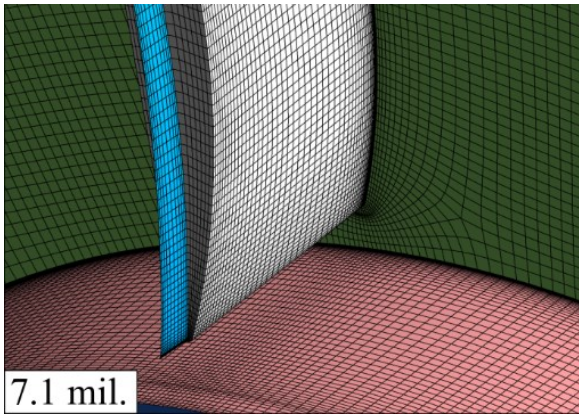

(d)

Fig. 3 (a) Mesh convergence study of $\sigma, \eta$ and $\Psi$, the mesh at impeller inlet for mesh No. 1 (b), No. 2 (c) and No. 4 (d).

Furthermore, the area covered by vapour at the suction side of the impeller is studied in order to determine when its shape and extent become spatially independent. As a consequence, the isosurfaces of the vapour pressure area are plotted for the four meshes in Fig. 4, where it can be observed that it is quite similar for the three finer of them, No. 2, 3 and 4 . Hence, the mesh No. 2, with 3.6 million elements is selected to be used throughout the present study.
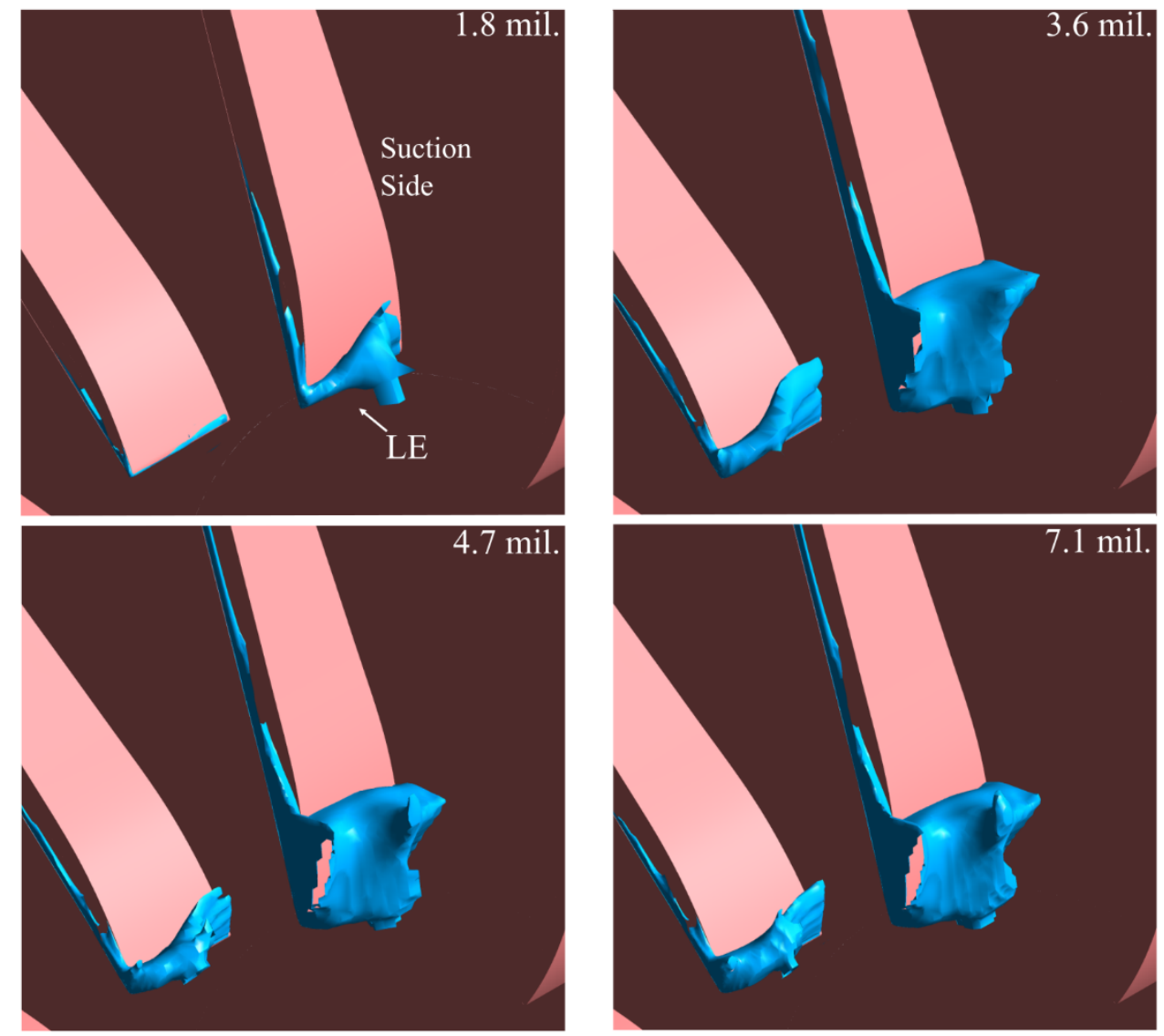

Fig. 4 Isosurfaces of vapour pressure area at the LE of the blades.

The effect of the impeller blades angular position relative to the volute has been examined. For this reason, the impeller section is rotated in three different angles $\left(\theta_{1}, \theta_{2} \& \theta_{3}\right)$ relative to the reference position, $\theta_{o}=0$, as shown in Fig. 5a, and the simulations are performed for all four positions at the same $\mathrm{OP}\left(\Phi=0.074 \cdot \Phi_{\mathrm{BEP}}\right.$ and $\left.\sigma=0.33\right)$.

The corresponding results for the $\eta, \sigma$ and $\Psi$ values are very slightly influenced by the exact impeller's position, as can be seen in Fig. $5 b$ (respective variations about 1.5\%, 2\% and $0.5 \%$ ). On the other hand, the vapour cavities developed close to the blades leading edge (Fig. 5c,d) exhibit similar characteristics but size differences, which are due to the non-axisymmetric 
pattern of the flow at the pump imlet, discussed in section 3, below. Consequently, the computational domain with the impeller at position $\theta=\theta_{0}$ is considered suitable and representative for the simulations of the present study.

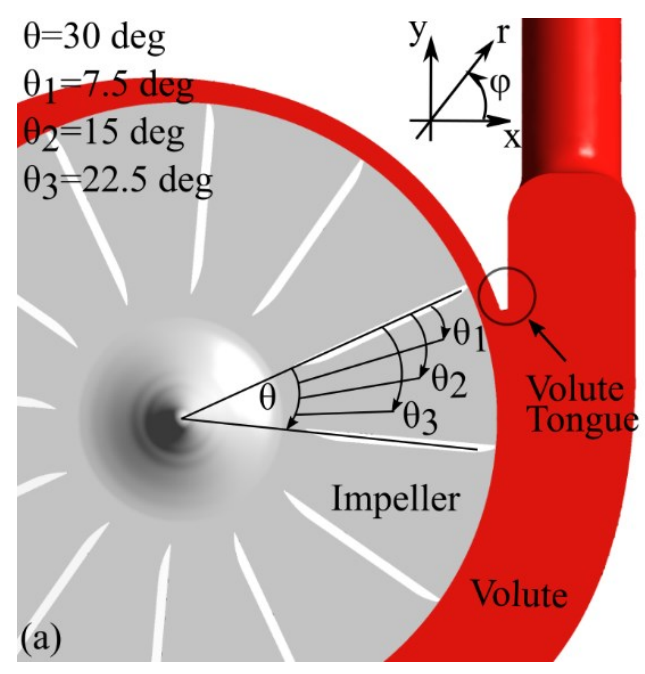

(c)

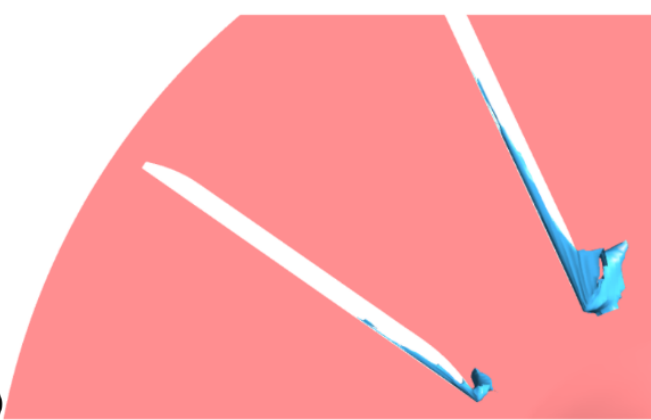

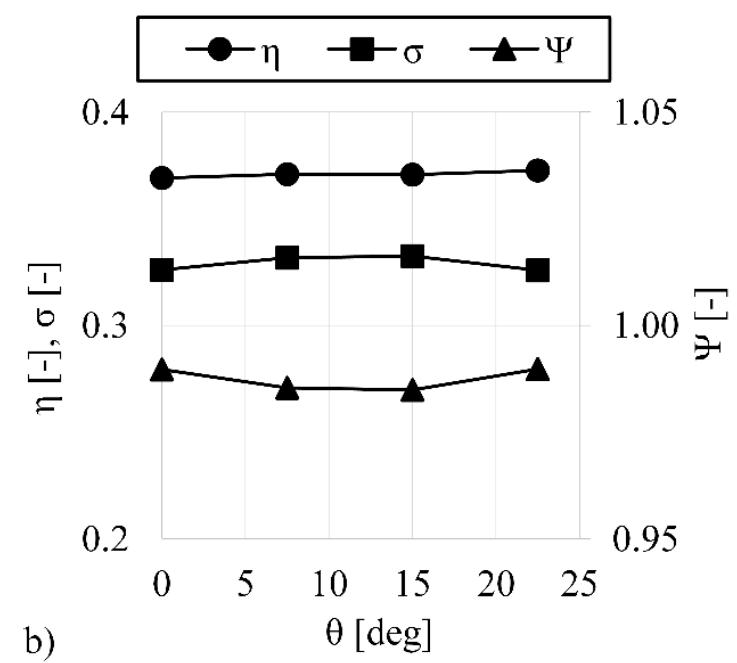

(d)

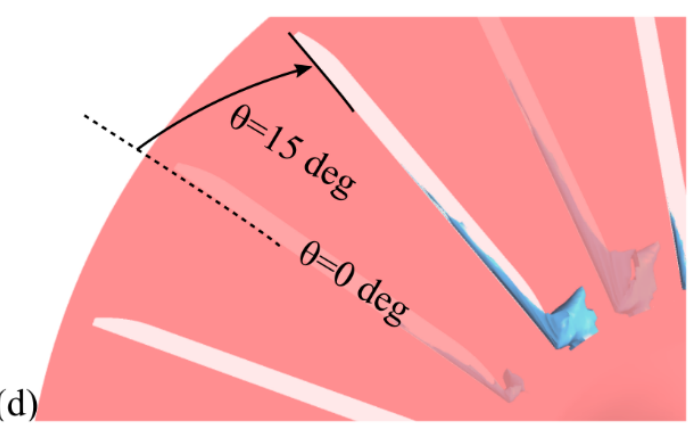

Fig. 5 Impeller relative angular position (a), and its effect on the resulting values of $\eta, \sigma$ and $\Psi$ (b) and on the cavitation development (c, d).

For the boundary conditions of the simulated domain (Fig. 1a), mass flow rate at the inlet and pressure outlet value are given from the values obtained during measurements. The absolute value of vapour pressure for the water was set to $p=2.8 \mathrm{kPa}$ that corresponds to $T=23^{\circ} \mathrm{C}$, the temperature that all the experiments were carried out. In this study, six $\Phi$ values were simulated for the derivation of $\Phi-\Psi$ and $\Phi-T$ curves, while four $\Phi$ values were selected for cavitation computations at various $\sigma$ values. The steady state approach is considered for all the simulations, with the use of the moving reference frame technique. More specifically, the domain that corresponds to the impeller remains frozen relative to the volute (frozen rotor approach), while the flow equations (in the impeller domain) are solved in the rotating reference frame. For this reason, interface surfaces are defined between the rotating and stationary frames, where the equations are transformed from the stationary to the rotating system of reference. It is noted that all the numerically obtained results of the present study illustrate the steady state converged solutions of the iterative numerical procedure. Second order of spatial discretization is selected for the pressure, momentum, volume fraction and turbulence. The algorithm converges when the normalized residuals of the flow equations are reduced below $10^{-4}$.

\subsection{Tip clearance modelling}

In order to study the effect of the blades tip clearance on the onset and development of cavitation, two more geometries are generated: one with a narrower tip with thickness $t_{t c}=0.5$ $\mathrm{mm}$, and one with a larger tip with $t_{t c}=1.5 \mathrm{~mm}$. The study includes two stages, i) the effect of tip clearance on the characteristic curves of the pump under single phase conditions, and ii) the comparison of pump performance for different tip clearance thicknesses under cavitating 
conditions. This requires the same $\sigma$ and $\Phi$ values, which creates additional difficulties to the simulation. On the one hand, the flow rate is set as inlet boundary condition and cannot be changed during the simulation. On the other hand, the cavitation parameter is a function of the calculated static pressure at the inlet and the total head of the pump (Eq. 13). Consequently, in order to achieve the same $\sigma$ value in all cases, the static pressure at the outlet boundary is regulated accordingly. This justifies the application of different boundary conditions at each tip clearance case.

\section{Experimental set up}

Experiments were carried out in a laboratory pump in the Engineering Department of Lancaster University, which is shown in Fig. 6. The water is pumped from the tank and passes through the suction valve before it enters the centrifugal pump that has a casing made from Plexiglas in order to visualize the flow inside the impeller. After the pump, the flow passes through the flowmeter and the discharge valve, which controls the flow rate, and returns to the main tank.

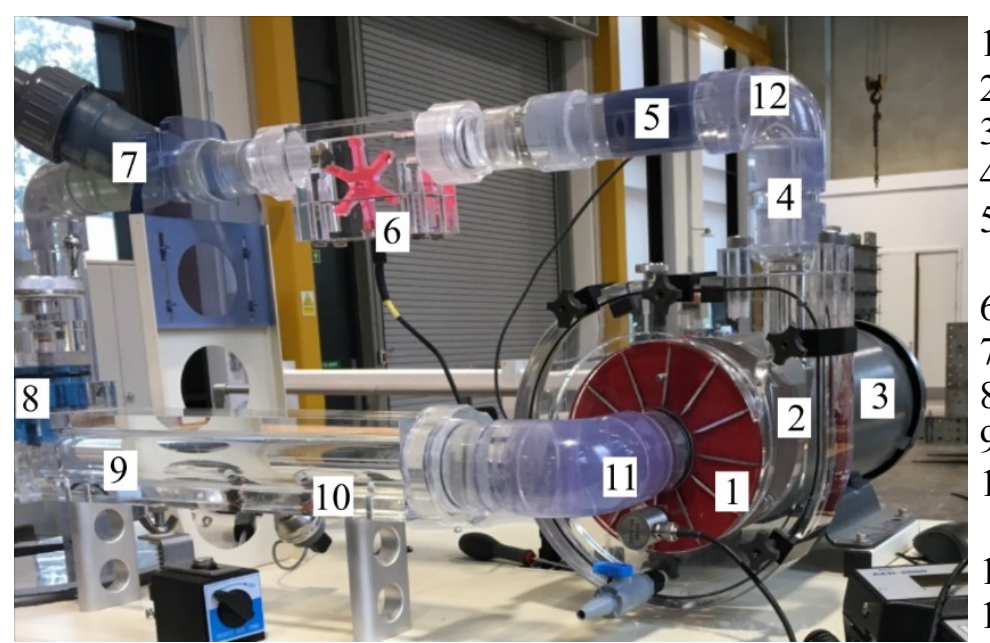

1. Impeller

2. Plexiglas Casing

3. Electric Motor

4. Discharge Pipes

5. Discharge Static Pressure Sensor

6. Flowmeter

7. Discharge Valve

8. Suction Valve

9. Suction Pipe

10. Suction Static Pressure Sensor

11. $90^{\circ}$ Elbow (1)

12. $90^{\circ}$ Elbow (2)

Fig. 6 Test rig at Lancaster University Laboratory.

For every Operating Point (OP) tested, the flow rate, the inlet and outlet gauge pressures, the electric power, and the rotational speed measurements are taken with sampling frequency and duration of $1 \mathrm{kHz}$ and 120 seconds, respectively. In order to study the inception of the initial bubbles and their development, the suction static pressure is progressively reduced by throttling the suction valve in order to reduce the $\sigma$ value, while properly regulating the discharge valve to retain the flow rate, according to $[69,70]$. This elaborate procedure is carefully carried out by hand, in order to regulate the flow rate with the desired accuracy (deviation below $1 \%$ along the entire $\sigma-\Psi$ curves) and to obtain repeatable measurements. Repeatability is verified for all tested impellers, as can be seen in the example of Fig. 7, where the measurements of three cavitation tests for the same flowrate $(\Phi=0.061)$ are plotted.

However, this method imposes a restriction to examine only partial flow rates, because for high flow rates the discharge valve is initially quite open and cannot be used to restore the flow rate after the throttling of the suction valve.

In the present study, flow visualization is used as a tool that confirms macroscopically the existence of bubbles, their onset as well as the shape, size and position of the vapour phase, and compares all the above with the corresponding numerical results. The experimental photos presented throughout this work are representative of the visually observed characteristics (location and shape) that the vapour phase exhibits during the $120 \mathrm{sec}$ of each corresponding measurement. As it is discussed in section 4.3.1, there are operating points where the vapour 
area exhibits strong attached cavitation characteristic, which keep its extent and position relatively unchanged with time.

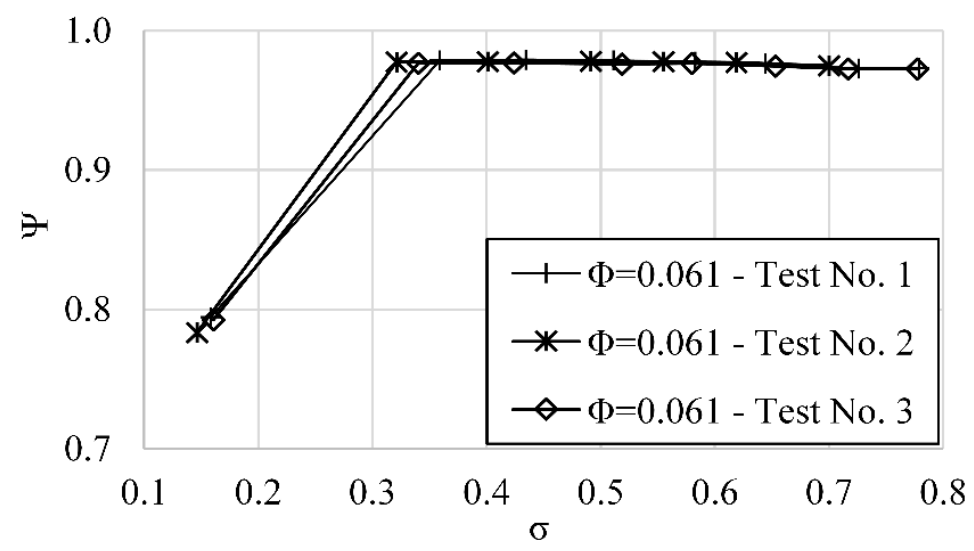

Fig. 7 Indicative repeatability of the experimental $\sigma-\Psi$ characteristic curves, for $\Phi=0.061$.

Moreover, it is possible that micro-cavities exist before optical visual inception, as well as outside of the limits of the visible two phase flow area. However, the available potential of the current visualization is considered. For each OP, the flow field inside the impeller is captured in a photo with the use of a stroboscope and a camera of DSLR type with $4752 \times 3168$ recorded pixels, CMOS image sensor and max shutter speed of $0.25 \mathrm{msec}$. In all tests the rotation speed is kept constant at $1800 \mathrm{rpm}$ and the fluid temperature at $23^{\circ} \mathrm{C}$. The tests are performed three times in order to ensure repeatability of measurements of the flow quantities, as well as of the cavitation inception and development. The total relative uncertainties, $f_{t, Y}$, for all the measured and calculated quantities are given in Table 3 . The uncertainties are calculated according to the relations given in [71], except of the efficiency uncertainty, which is obtained by Eq. 18 .

Table 3 Total relative uncertainty for all the measured and calculated quantities.

\begin{tabular}{ccccccc|cccccc}
\hline \multicolumn{7}{c|}{ Measured Variables } & \multicolumn{4}{c}{ Calculated Variables } \\
\hline $\boldsymbol{Y}$ & $\boldsymbol{Q}$ & $\boldsymbol{p}_{\text {Suc }}$ & $\boldsymbol{p}_{\text {Dis }}$ & $\boldsymbol{T}$ & $\boldsymbol{\Omega}$ & $\boldsymbol{P}_{\boldsymbol{e l}}$ & $\boldsymbol{\Phi}$ & $\boldsymbol{\Psi}$ & $\boldsymbol{\sigma}$ & $\boldsymbol{\eta}$ & $\boldsymbol{H}_{\text {tot }}$ & $\boldsymbol{N} \boldsymbol{P S H}$ \\
\hline $\pm f_{t, Y}[\%]$ & 0.6 & 0.3 & 0.3 & 1 & 0.1 & 1.2 & 0.6 & 0.6 & 0.8 & 1.5 & 0.6 & 0.6 \\
\hline
\end{tabular}

$$
f_{t, \eta}= \pm \sqrt{\left(\frac{F_{t, H_{t o t}}}{\overline{H_{t o t}}}\right)^{2}+f_{t, Q}{ }^{2}+f_{t, P_{e l}}^{2}}
$$

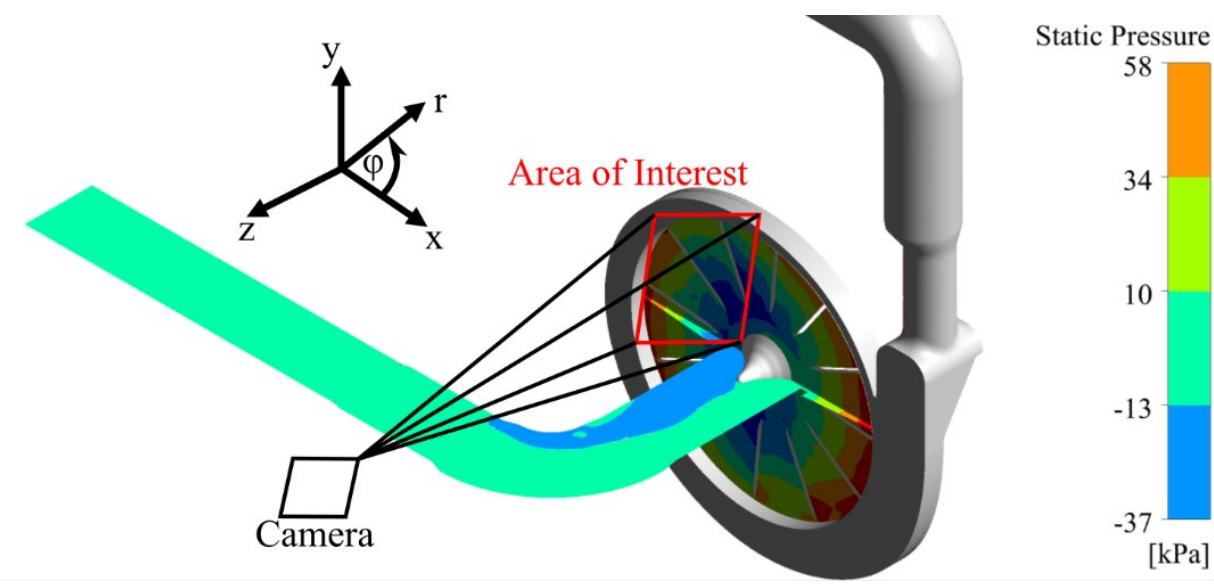

Fig. 8 The non-axisymmetric flow field at the suction and the impeller of the pump. 
The $90^{\circ}$ bending pipe upstream of the impeller inlet (No. 11 in Fig. 6) and the volute of the pump disturb the axial symmetry of the flow and creates an additional pressure drop. The nonaxisymmetric pressure field at the impeller inlet is shown in Fig. 8 and justifies the fact that during the experimental tests cavitation inception appears first in the left side $\left(90^{\circ}<\varphi<180^{\circ}\right)$ of the impeller eye. For this reason, the camera is fixed at the left side of the impeller, as it is also depicted in Fig. 8, in order to take comparable pictures for all examined cases. For the same reason, the validation and the presentation of the two phase flow results focuses on this region of the impeller. It is noted, that a second bending pipe is installed at the exit of the pump (No. 12 in Fig.6), downstream of the impeller, which creates additional friction losses, similar to the elbow at the suction side (No. 11 in Fig.6).

\section{Results and discussion}

\subsection{Validation of the numerical results}

The validation methodology includes the comparison of the performance curves at both single and two phase flow conditions in order to ensure the reliability of the modelling methodology. The calculated and measured head, efficiency and power characteristic curves are given in Fig. 9.

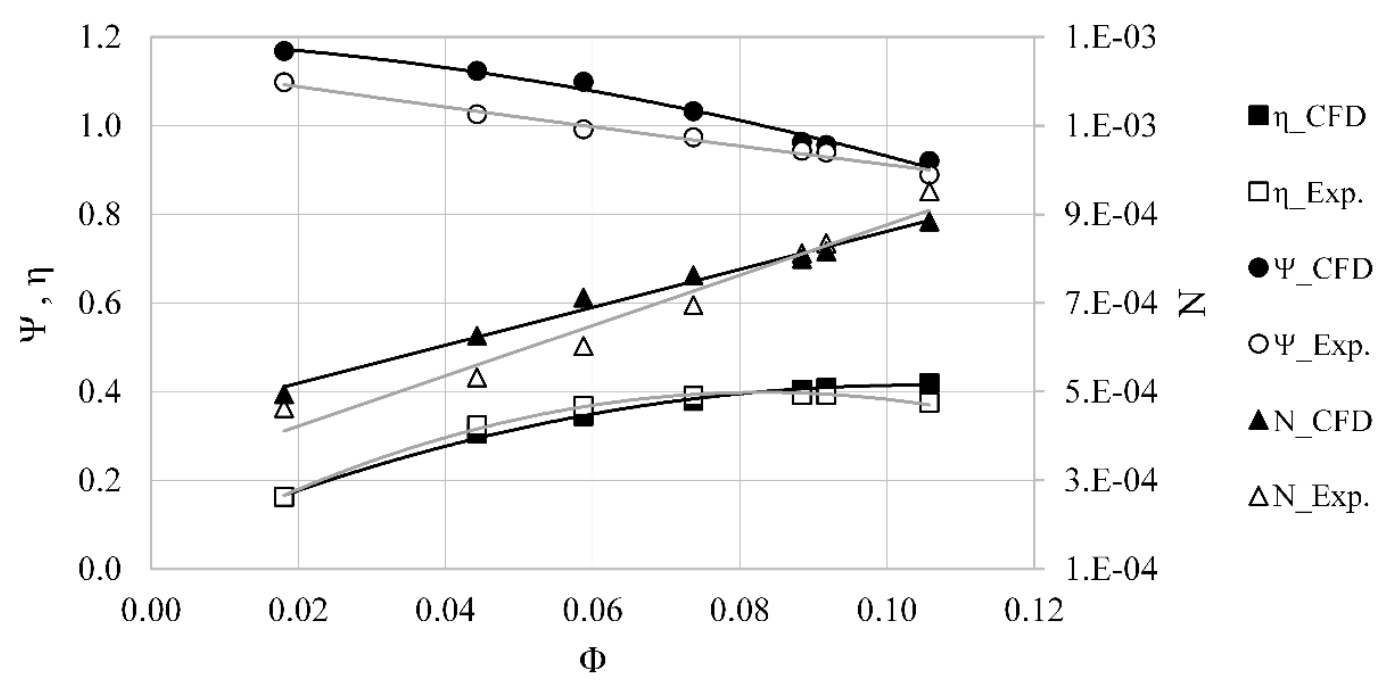

Fig. 9 Experimental and numerical curves under non cavitating conditions.

All the curves exhibit the qualitative trend of centrifugal pump impellers in this specific speed area, i.e. $\Psi$ is a monotonically decreasing function of $\Phi, N$ increases with $\Phi$ and $\eta$ has one global maximum. Very good agreement for all variables is achieved close to the BEP of the pump $(\Phi=0.091)$. The differences increase at partial loading, where the CFD model overpredicts at the same degree both the total head and the power, resulting in very good agreement of the efficiency characteristic curves (Fig. 9). The existence of pipe bends at the suction and the discharge pipe of the pump (Fig. 6) may be responsible for this difference. The $90 \mathrm{deg}$. turn of the fluid creates secondary flows that disturb the fully developed flow pattern for a few pipe diameters downstream. This, in turn, affects the uniformity of the static pressure field at the cross section of the pipe where the pressure sensors are installed, especially that located downstream of the discharge bend (Fig. 6), and hence, the accuracy of the pump head measurements. It is noted that the computational domain includes the geometry of both pipe bends (Fig. 1), therefore, a better agreement should have been achieved. Nevertheless, the total relative error remains below $10 \%$, and this systematic deviation does not affect much the further 
study of cavitation conditions of the pump, which are governed by the flowrate and the static pressure at the suction side.

The experimental and numerical results for the corresponding $\sigma-\Psi$ correlations are plotted in Fig. 10. At first, the deviation of $\Psi$ values in the non-cavitating region of $\sigma$, increases as the flow rate becomes lower, as discussed previously in Fig. 9. In the cavitating region, between the last two OPs measurements, the slightest change of valve position resulted in a drastic drop of suction pressure and the total head is collapsed (see also [72]). On the other hand, in the numerical analysis it is possible to simulate the flow conditions between these two lowest experimental $\sigma$ values, as shown in Fig. 10. The values of $\Psi$ exhibit barely noticeable differences, albeit the addition of the two-phase model. The experimental value of minimum $\sigma$ value, $\sigma_{F C}$ is systematically higher compare to the numerical one, similarly to previous published results $[19,44,65,70,73]$.
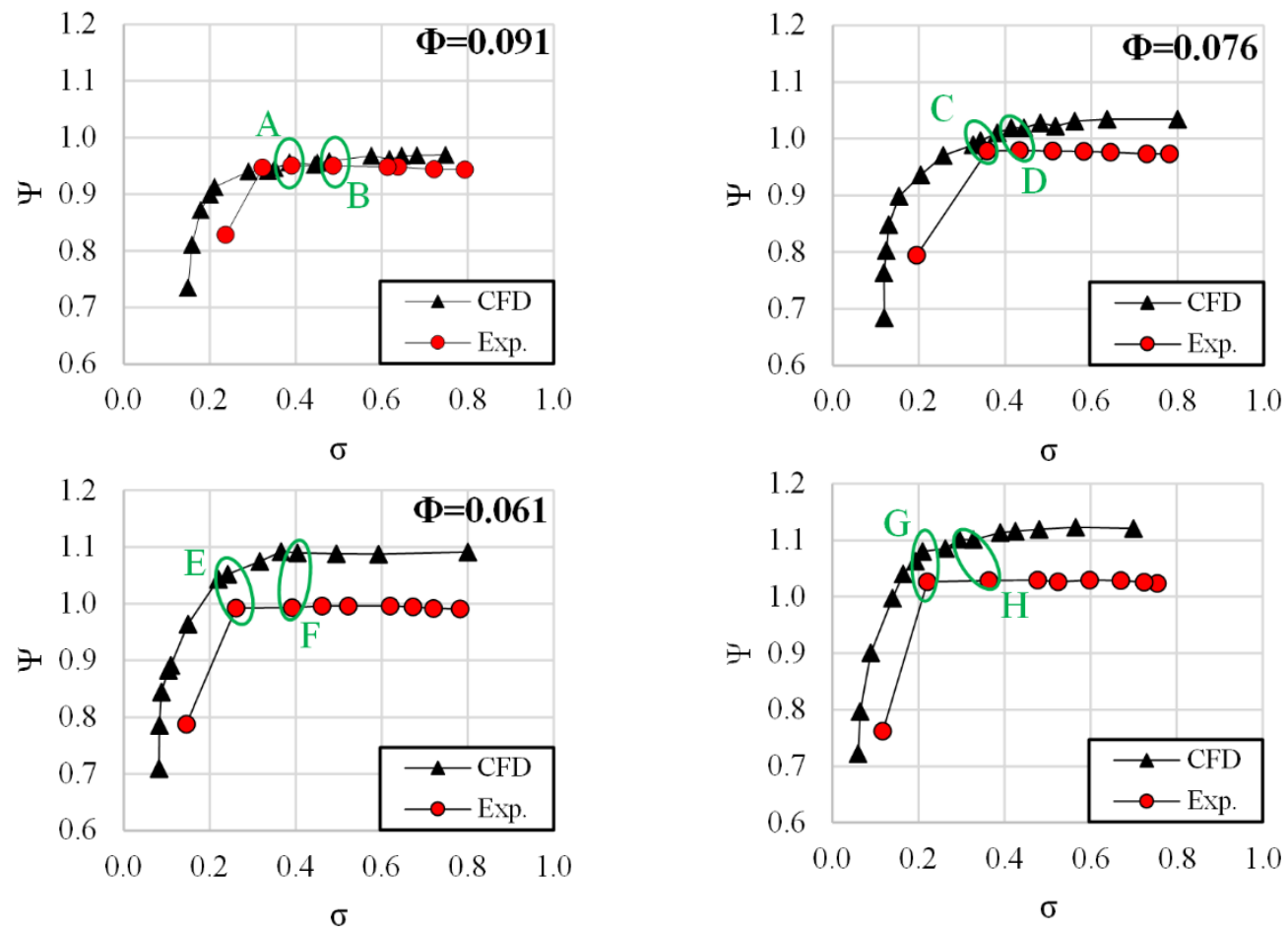

Fig. 10 Experimental and numerical $\sigma-\Psi$ curves for different $\Phi$ values.

As it was presented in studies that included a parametric analysis regarding the effect of non-condensable gas $[33,74]$, the effect of non-condensable gases that is not taken into account in the current simulation, can cause the head collapse to happen earlier, namely at higher $\sigma$ values. Additional factors that may be responsible for the deviation between measured and computed $\sigma_{F C}$, are the values given to the vaporization and condensation constants of the cavitation model, the geometry inaccuracies, especially at the tip clearance area, and the fact that the impeller-volute dynamic interactions are not modelled here. In view of these uncertainties, the agreement in all the cases of Fig. 10 is quite satisfactory. The points A to $\mathrm{H}$ depicted in this figure will be discussed in section 4.3 below.

\subsection{Performance under non-cavitating conditions}

The calculated characteristic curves for $\Psi, \eta$ and $N$ of the pump for the three tip clearance cases are plotted in Figs. 11 and 12. Here, $\Psi$ value increased with the decrease of tip clearance thickness and was maximized at the case with the minimum tip clearance, due to the minimization of leakage flow passage along with the tip losses and vortex recirculation similarly to $[64,75-77]$. Similar behavior follows the efficiency of the pump due to the increase of the tip clearance losses. 


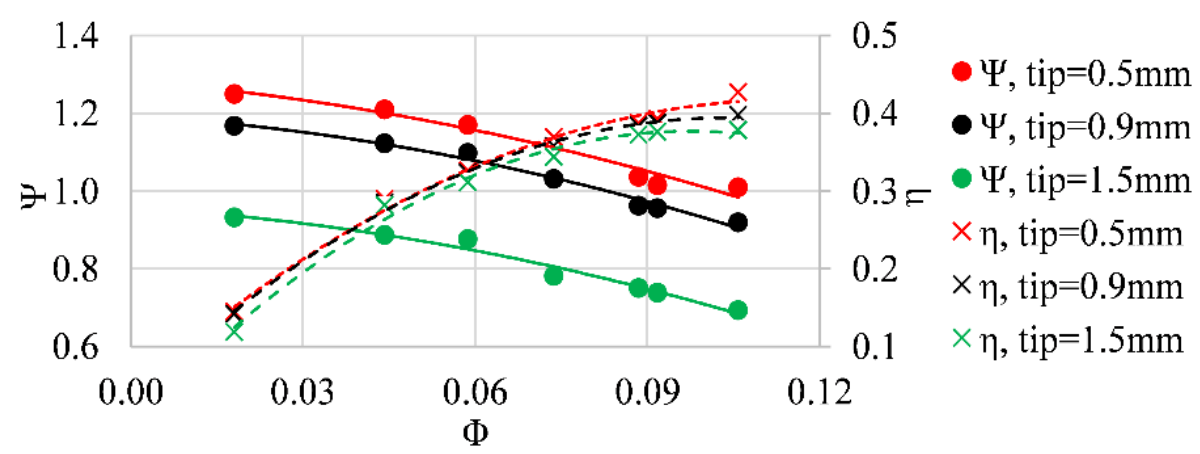

Fig. 11 Head and efficiency values for the three tip clearance cases modeled.

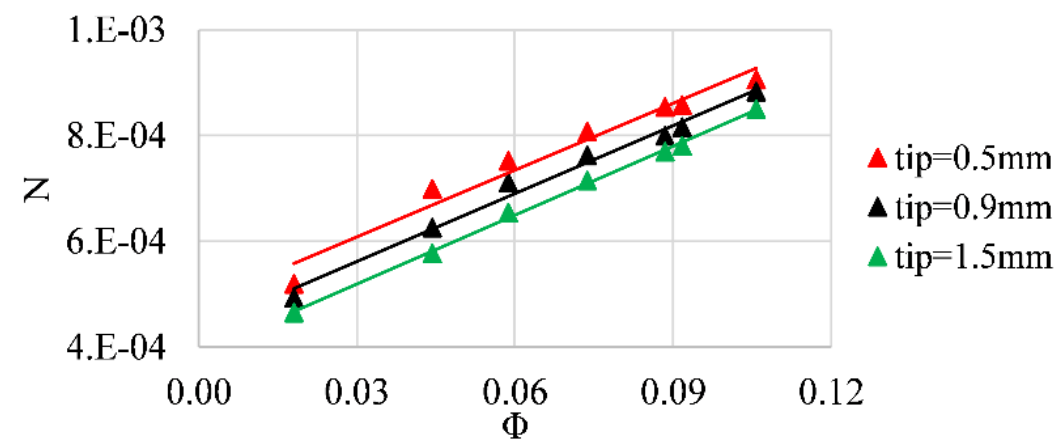

Fig. 12 Power values for the three tip clearance cases modeled.

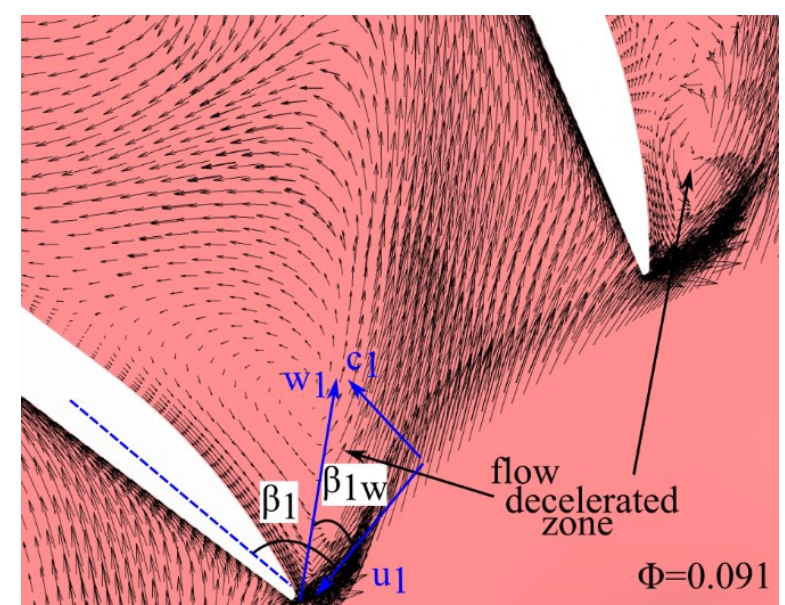

Fig. 13. Relative velocity vectors at the LE of the blades.

The relatively low efficiency of this pump ( $40 \%$ at BEP) shown in Fig. 9 is mainly justified from the non-hydrodynamic design of the impeller's blades and the absence of shroud. As can be seen in Fig. 13, the $90^{\circ}$ inlet angle of the blades results in a high incidence angle, $i\left(i=\beta_{1^{-}}\right.$ $\left.\beta_{I w}\right)$, of the relative flow velocity, $w_{l}$, that causes decelerated and flow separation zones at the blade inlet [78], even at $\Phi_{B E P}$ OP. Under such flow conditions, the recirculation appears at the low pressure side of the blades. The relative velocity vectors demonstrate the poor impeller design and the low efficiency measured and computed. In the same figure is also observed the non-axisymmetric effects at the recirculation areas that have different shape between two successive blades. 


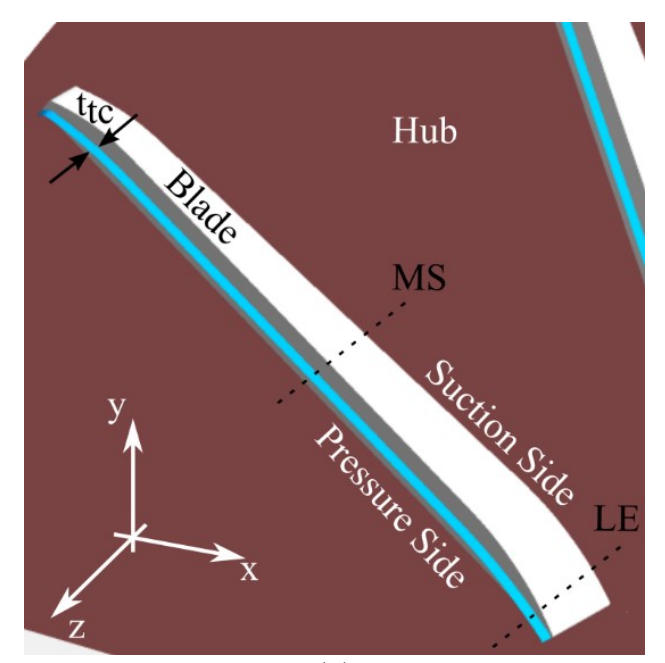

(a)

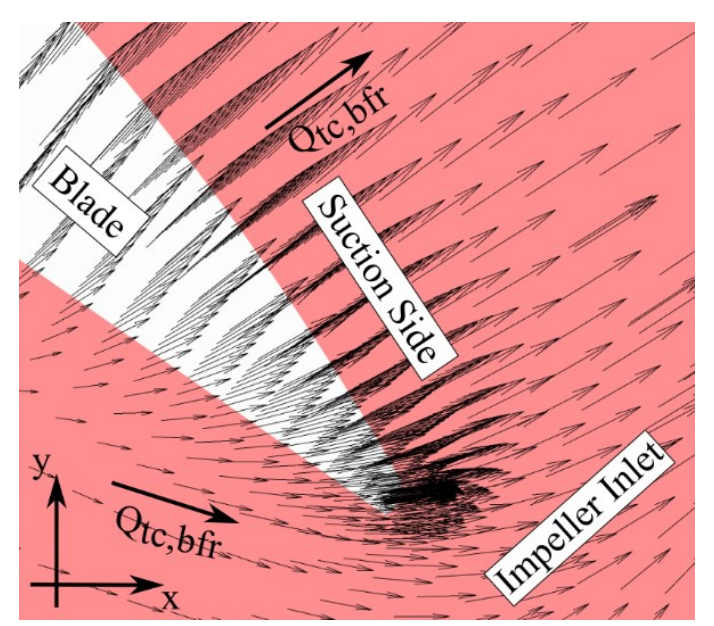

(b)

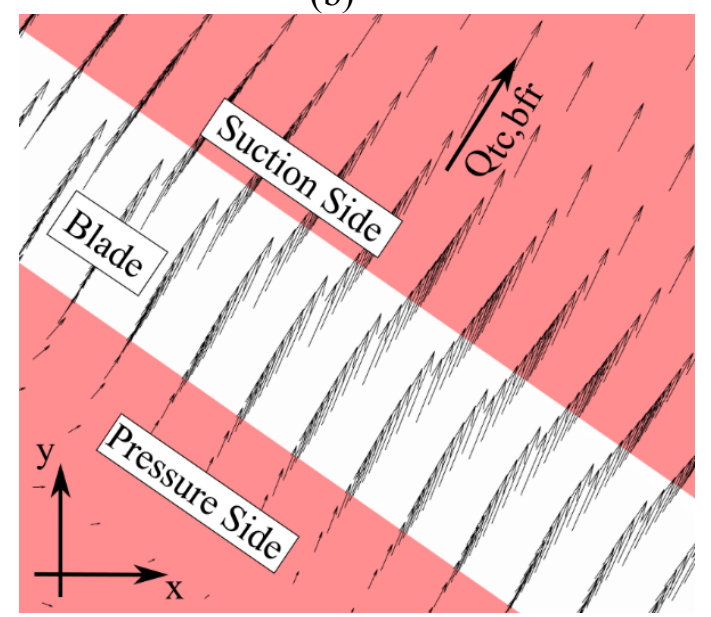

(d)

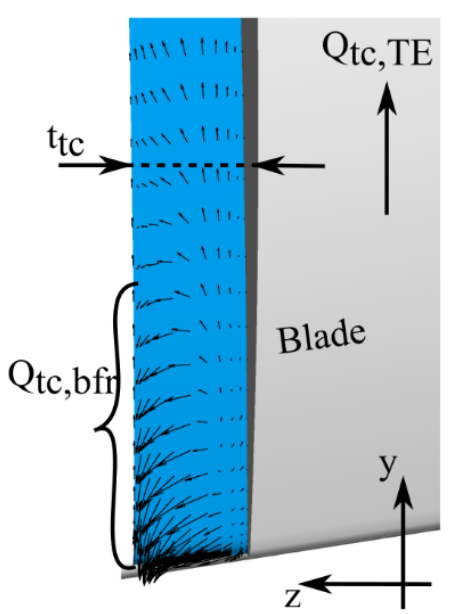

Impeller Inlet

(c)

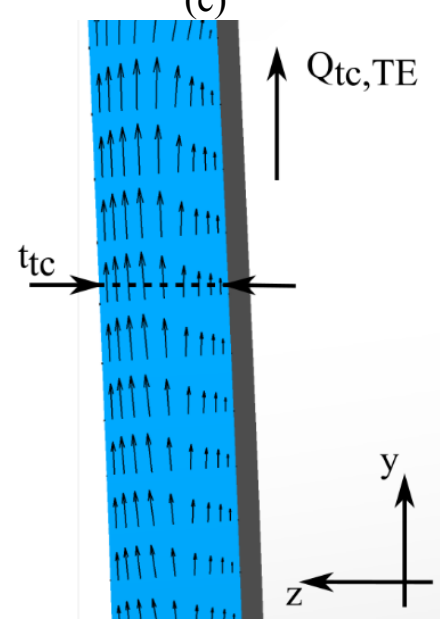

(e)

Fig. 14 (a) MS and LE area of the blade, relative velocity vectors at $\Phi=0.091$ at the original tip clearance geometry $\left(t_{t c}=0.9 \mathrm{~mm}\right)$ for (b) $\mathrm{A}_{1}, \mathrm{LE}$, (c) $\mathrm{A}_{2}, \mathrm{LE}$, (d) $\mathrm{A}_{1}, \mathrm{MS}$, (e) $\mathrm{A}_{2}, \mathrm{MS}$.

A second mechanism that affects the overall efficiency and the cavitation behavior of the pump is the tip clearance flow developed between the rotating blades tip and the stationary casing. In order to depict tip effects on the flow, the relative velocity vectors are plotted in Fig. 14 in two areas, i) at the blade LE and ii) at the midspan (MS) of the blade, and on two different planes; the plane $A_{1}$ along the tip (Fig. 14b,d), and the meridional plane $A_{2}$ (Fig. 14c,e). 
According to the results of Fig. 14, the flow at the tip consists of two components; i) the main part that is directed towards the suction, low pressure side of the blade and the inlet of the impeller and forms backflow recirculation $\left(Q_{t c, b f r}\right)$ and ii) a part that flows towards the trailing edge, $Q_{t c, T E}$. The latter is observable only on $\mathrm{A}_{2}$ planes, however backflow recirculation is more significant since it dominates flow conditions of the flow path. The only exception is the flow at the MS of the blade at $\mathrm{A}_{2}$ plane where this component of the relative velocity vectors flows towards the TE. A noteworthy observation that can be seen in Figs. 14b\&c is that backflow recirculation at the LE area may alter to backflow cavitation when machine operates at such conditions that favor two phase flow formation into the tip clearance. As a consequence, tip clearance recirculation could play significant role to the cavitation onset and development.

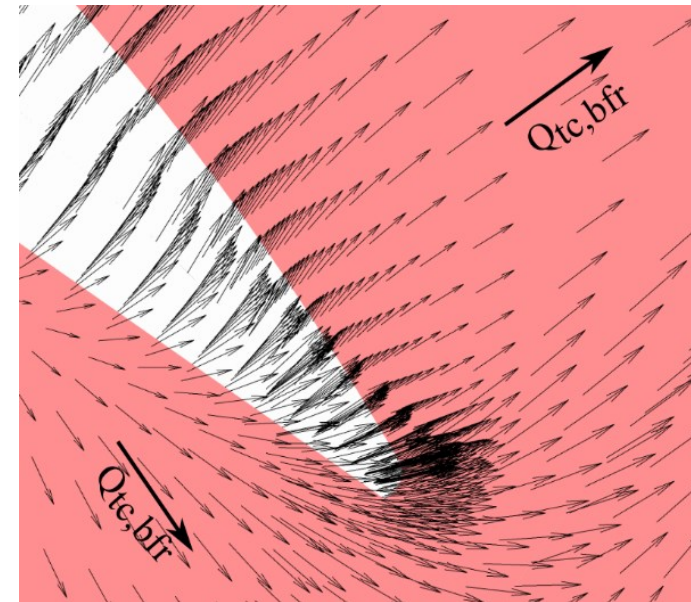

(a)

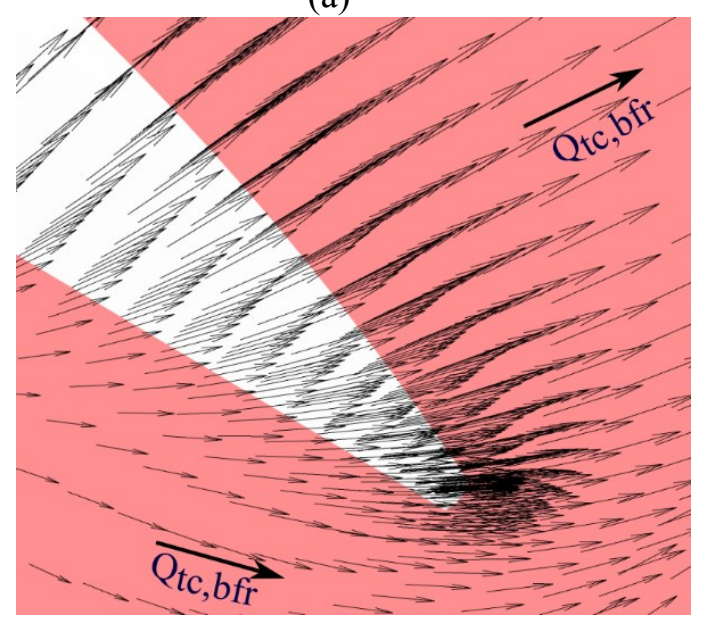

(c)

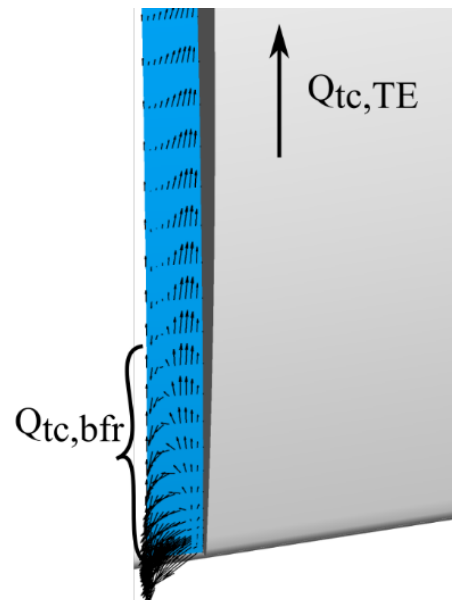

(b)

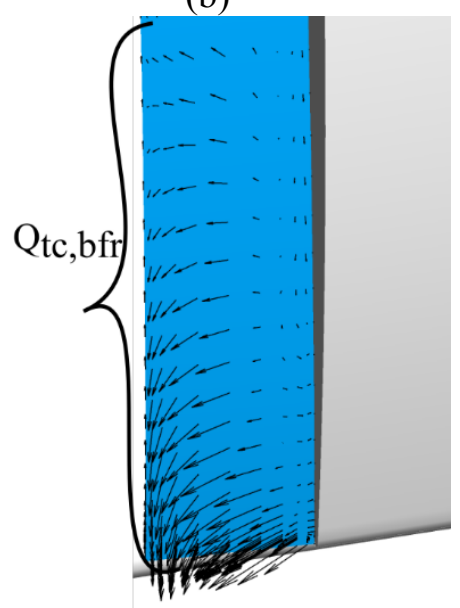

(d)

Fig. 15 Relative velocity vectors at $\Phi=0.091$ for (a) $A_{1}, t_{t c}=0.5 \mathrm{~mm}$, (b) $\mathrm{A}_{2}, t_{t c}=0.5 \mathrm{~mm}$, (c) $\mathrm{A}_{1}$, $t_{t c}=1.5 \mathrm{~mm}$, (d) $\mathrm{A}_{2}, t_{t c}=1.5 \mathrm{~mm}$.

For this reason, the effect of tip clearance size $t_{t c}$ at the backflow phenomenon is also examined, and the results for $\Phi=0.091$ are presented in Fig. 15 only for the LE area. As was expected, the direction of back flow recirculation, $Q_{t c, b f r}$, remains similar with the original geometry (Fig. 14b,c) for both larger and smaller $t_{t c}$ values tested, however its strength is analogous to the tip clearance (Fig. 15) Especially at $A_{2}$ plane, the intensity and the size of the recirculation vortex, appears to be affected significantly by the tip clearance thickness (Fig. $15 \mathrm{~b}, \mathrm{~d}$ and 14b), and both enlarge with the increase of $t_{t c}$. 


\subsection{Performance under cavitating conditions}

The performance of the pump under cavitating conditions is studied in three stages. Firstly, an appropriate vapour volume fraction, $\alpha_{v}$ for the optical representation of the interface of the two phase flow must be selected. Then, the computational results for various flow rates, and for three $\sigma$ OPs, one at the onset of the phenomenon, one at intermediate cavitation stage and one at the lowest $\sigma$ value, are presented and compared with the flow visualization results captured by the camera. Finally, the computational results of the flow at the tip clearance during cavitation inception and development are presented and discussed.

\subsubsection{Cavitation inception and development.}

In order to investigate the two phase flow conditions inside the impeller, the photos obtained with the DSLR camera are compared directly with the computational results at the OPs depicted with the capital green letters in Fig. 10. The area that encloses the cavities in the simulation results is visualized by the use of isosurfaces curves of vapour volume fraction, $\alpha_{v}$ (Fig. 16b). From the indicative comparative results of Fig. 16 it can be deduced that the isosurface with $\alpha_{v} \approx 0.5$ provides a satisfactory representation of the macroscopically visible vapour area, so as to agree with the corresponding experimental photo. The same value is also adopted in other works [79-82]. This selection does not mean that a lower value of vapour area would be incorrect, however the camera limitations could not confirm the existence of cavitaties at smaller $\alpha_{\mathrm{v}}$.

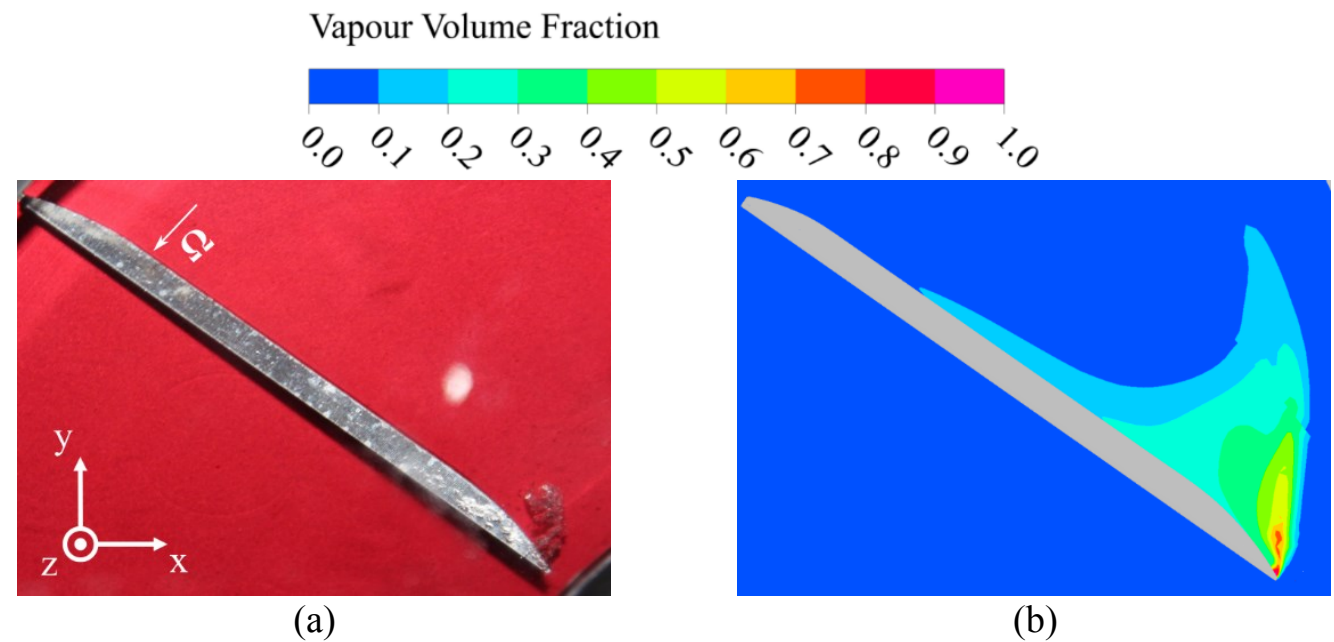

Fig. 16 (a) Flow visualization and (b) Vapour volume fraction at OP C (Fig. 10).

The experimental flow visualization results are compared with the numerical results obtained for $\alpha_{v}=0.5$ (blue color areas) in Fig. 17, for all the OPs A to H, as they are marked in Fig. 10. The values of $\sigma$ and $\Psi$ that define the corresponding experimental and numerical OPs, are selected as close as possible in order to be comparable.

As expected, in both the experimental and computational results cavitation appears at the suction side of the blade close to the LE. Moreover, the numerical results in Fig. 17 show that cavitation initiated in the tip clearance region of the blade, at the same time (e.g. point $\mathrm{H}$ ), or even earlier (point F). This may also happen in the real impeller, but cannot be observed in the corresponding photos due to the light reflection at the blade side surface. In all cases, the size of the two phase flow becomes larger when the $\sigma$ value reduces and approaches the head collapse limit. In addition, the most intense cavitation appears at E and G OPs (Fig. 17), which are those with the lowest $\sigma$ value $(\sim 0.2$, see Fig. 10$)$. In these cases the cavity occupies significant span of the tip clearance close to the LE of the blade. In most cases of Fig. 17 the simulated two-phase flow areas agree in general with the corresponding visual observations and pictures taken. The few differences noticed are mainly due to the difficulty of the model to predict the dynamic characteristics of the phenomenon. 


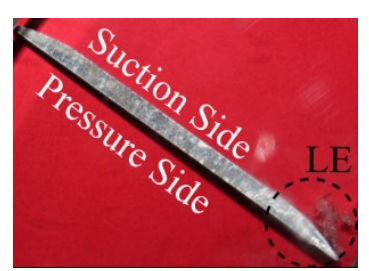

A

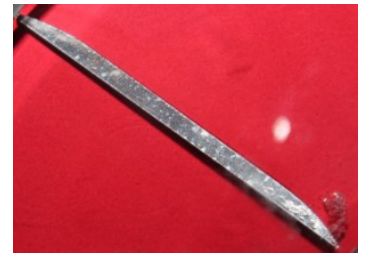

$\mathrm{C}$

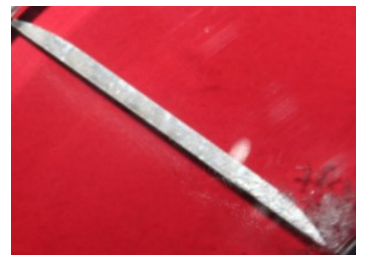

$\mathrm{E}$

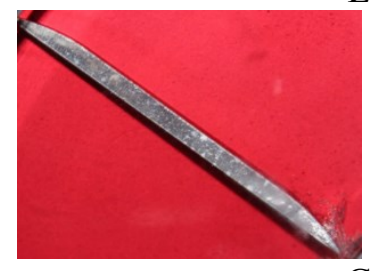

G

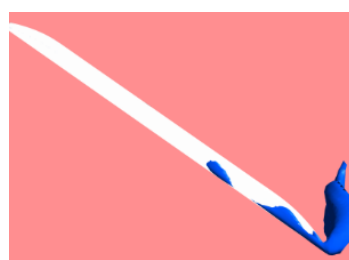

A

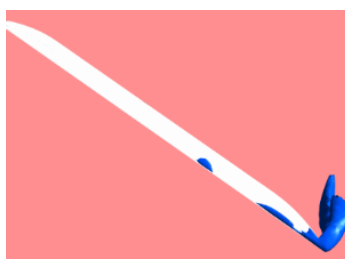

C
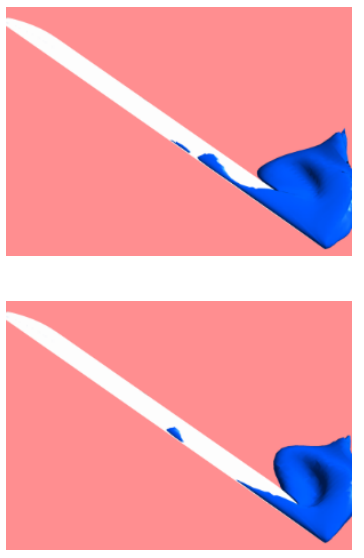
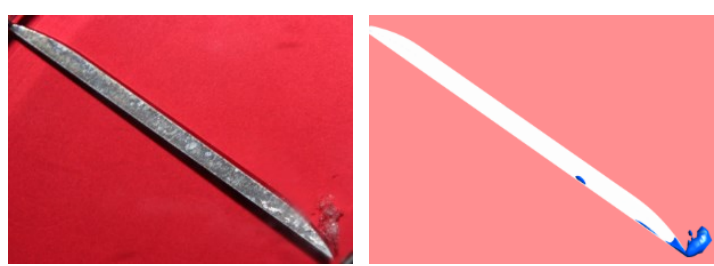

$\mathrm{B}$
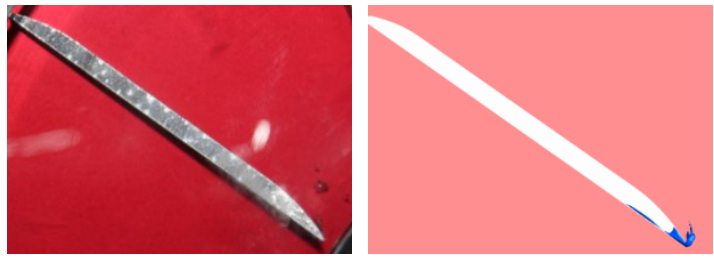

$\mathrm{D}$
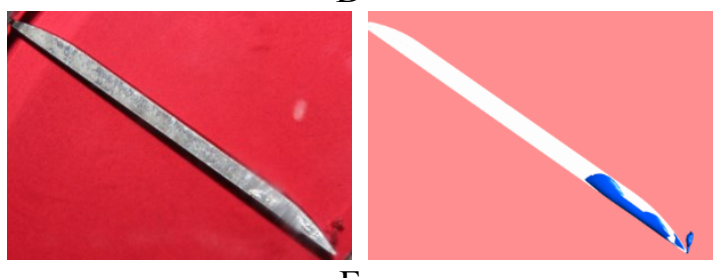

$\mathrm{F}$
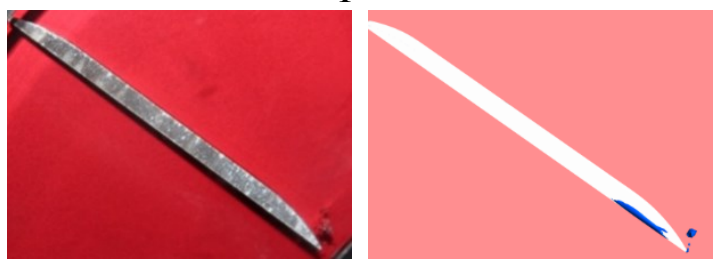

$\mathrm{H}$

Fig. 17 Experimental and numerical two phase flow results for various $\Phi$ and OP marked in Fig. 10: Points B, D, F, H are close to cavitation inception, and points A, C, E, G are at smaller $\sigma$ values (developed cavitation).

Although cavitation is an inherently unsteady mechanism, the macroscopic two-phase flow formations presented in this section exhibit a combination of an almost steady and a transient part, the appearance and relative size of which is related with the suction conditions (value of $\sigma$ ). When the pump operates at relatively higher $\sigma$ values, such as in the case of OPs B, D, F and $\mathrm{H}$ (Fig. 17), the bubble formations is unquestionably dynamic: the bubbles are created randomly and re-liquified abruptly, showing a typical travelling bubble cavitation type [83]. Consequently, a steady state MRF simulation is unable to predict accurately this strongly unsteady behavior, and the numerically obtained results underestimate the actual extent of vapour area, as it can be observed especially for the OPs B and D (Fig. 17).

On the other hand, when the study focuses on lower $\sigma$ values, such as in OPs A, C, E and G (Fig. 17), the vapour formations in the impeller contains an almost steady attached cavity that does not change significantly over time, and a second area at the downstream limits and beyond of the attached cavity, which consists of vapour bubbles that exhibit unsteady behaviour. The appearance of the attached cavity is the result of the flow separation that appears in the relative velocity vectors of Fig. 13, due to the increased incidence angle of the present impeller. As a result, in the OPs A, C, E and G of Fig. 17, the steady part of the two phase flow becomes stronger and the model predictions regarding the extent and the shape of the two-phase flow area are improved.

For all the above cases, the photos presented in Fig. 17 to 19 are carefully selected to be as representative as possible of an 'average' picture of the flow field observed during the flow visualization experiments. 


\subsubsection{Severe cavitation}

The experimental and numerical results of the flow in the impeller at the point when $\Psi$ collapses are compared in Fig. 19 for the four $\Phi$ values tested. At these operating conditions the cavities block the entire path between the impeller blades, and the visual observations reveal that the flow becomes very unsteady and exhibits intensive recirculation and separation regions. In order to discuss in more detail the complex flow conditions during severe cavitating conditions, the results for a particular flowrate $(\Phi=0.061)$ are shown separately, in Fig. 18, where different vapour formations coexist in the impeller flowpath:

i. Attached vapour cavities at the suction side of the blade, forming a homogenous single bubble.

ii. Cloud bubble cavitation at and beyond the limits of the attached cavitation area.

iii. A dynamic wake of vapour bubbles in the flowpath downstream of the cloud bubble area.

iv. Tip cavitation in the blade leakage area, which causes the backflow cavitation phenomenon.

The aforementioned types can be also observed in all experimental photos of Fig. 19.

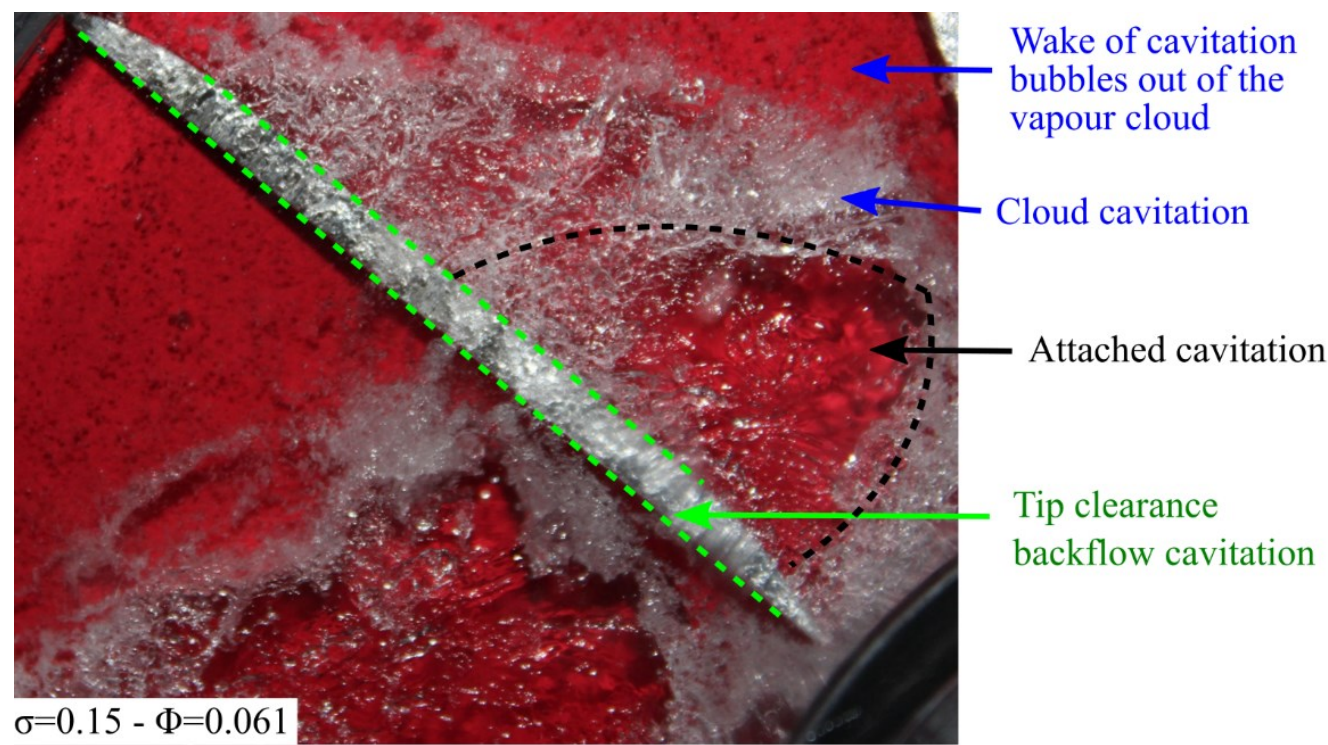

Fig. 18 Various cavitation types that can be observed during heavy cavitating conditions.

The non-periodic flow can be also observed at this OP for all flowrates, where the extent of the two phase region is different at neighboring flow passages. This behavior is sufficiently reproduced in the corresponding numerical results shown in Fig. 19. Furthermore, the numerically obtained results of Fig. 19 are not able to predict the wake of the cloud area that presents unsteady characteristics, since the steady state simulations suppress this part of twophase flow area.

Although the agreement between the simulation and the test results for the two phase flow area is very satisfactory, it takes place at similar $\Psi$ but different $\sigma$ values, as discussed above in section 4.1 and shown in Fig. 10. More specifically, the numerical $\sigma$ values are lower than the corresponding experimental ones, showing that the model predicts the total head breakdown with a delay. Finally, at the lowest flow rate $(\Phi=0.046)$, the two phase flow extends from the LE to the TE and all the area from the suction to the pressure side of the next blade (Fig. 19). The cavitation area decreases with the increase of $\Phi$, and for higher flow rates $(\Phi=0.061$, $\Phi=0.076 \& \Phi=0.046$ ) it covers smaller blade span. This is due to the fact that the minimum $\sigma$ value is higher for higher $\Phi$ values (Fig. 10), and as a consequence, the phenomenon exhibits narrower characteristics. The extent of the cavities, especially at the minimum $\sigma$ point, affect the ability of the blade to convert mechanical to fluid power, and hence, $\Psi$ collapses. 


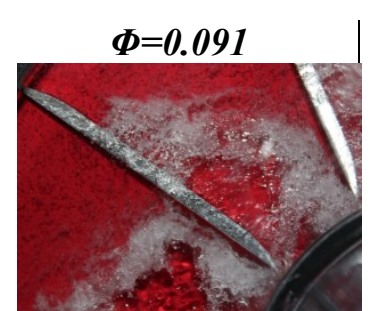

$\sigma=0.24, \Psi=0.83$

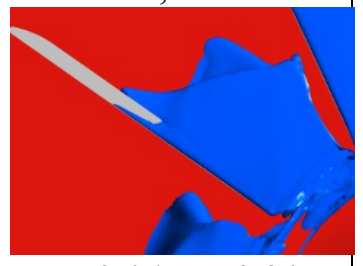

$\sigma=0.16, \Psi=0.81$

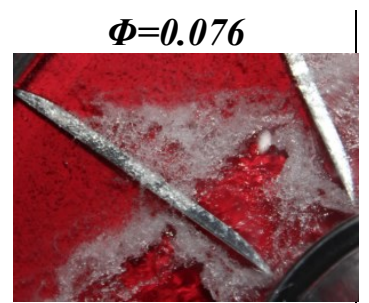

$\sigma=0.19, \Psi=0.79$

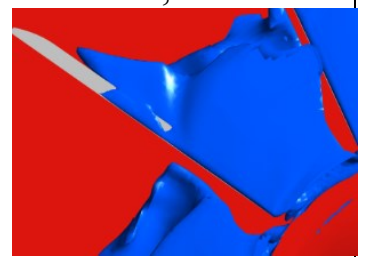

$\sigma=0.12, \Psi=0.80$

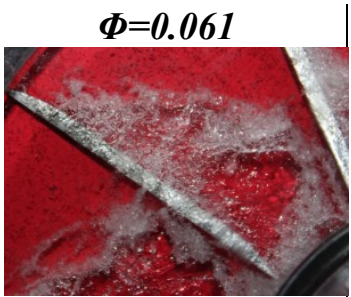

$\sigma=0.15, \Psi=0.79$

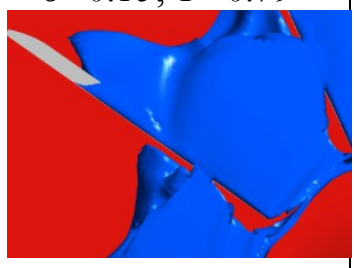

$\sigma=0.08, \Psi=0.79$

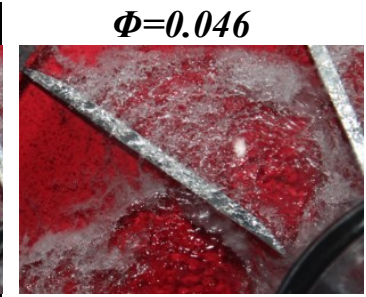

$\sigma=0.12, \Psi=0.76$

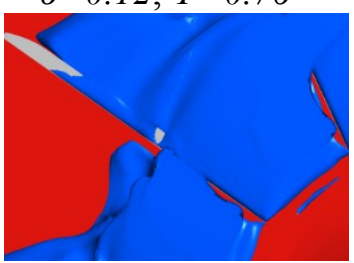

$\sigma=0.06, \Psi=0.80$

Fig. 19 Flow visualization (first line) and numerical results (second line) of the extent of cavitation flow inside the impeller at $\Psi$ collapse point, for various $\Phi$ values.

\subsubsection{Tip clearance effect on cavitation}

The performance of the present pump is significantly affected by the absence of shroud at the impeller. In Section 4.2, it was concluded that backflow recirculation from the tip is important close to the LE of the blade. This could strongly affect cavitation development at lower $\sigma$ values and possibly create conditions that favor backflow cavitation. For this reason, the analysis of tip clearance effect on cavitation starts with the comparison of the relative velocity vectors at the LE for three different OPs; $\sigma_{l}=0.62$ is close to the onset of the first bubbles, $\sigma_{2}=0.34$ at an intermediate stage of development, and $\sigma_{F C}=0.16$ corresponds at the total head breakdown for $\Phi=0.091$. The results are compared in Fig. 20, together with the static pressure contours. It is noted that all pressure contours presented in this study depict the relative to atmospheric (gauge) pressure. The static pressure decreases gradually in the entire LE region with the reduction of $\sigma$ value, while cavitation grows and captures larger volumes at the suction side of the blade, at the impeller inlet and in the tip clearance area (Fig. 20). As expected, at $\sigma_{F C}$ the low pressure cavitating flow (absolute pressure of $2.8 \mathrm{kPa}$ ), dominates the entire tip clearance.

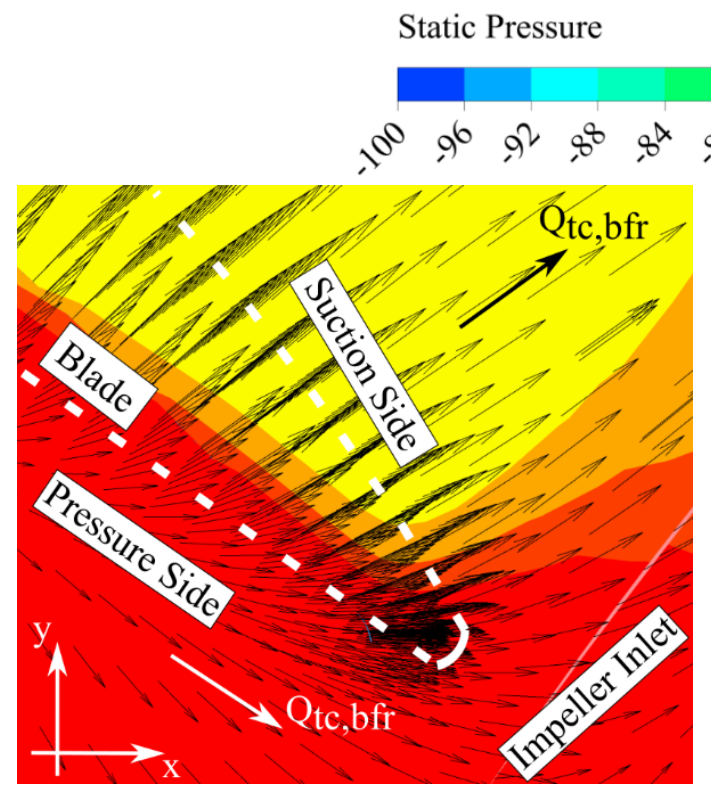

(a)
$[\mathrm{kPa}]$

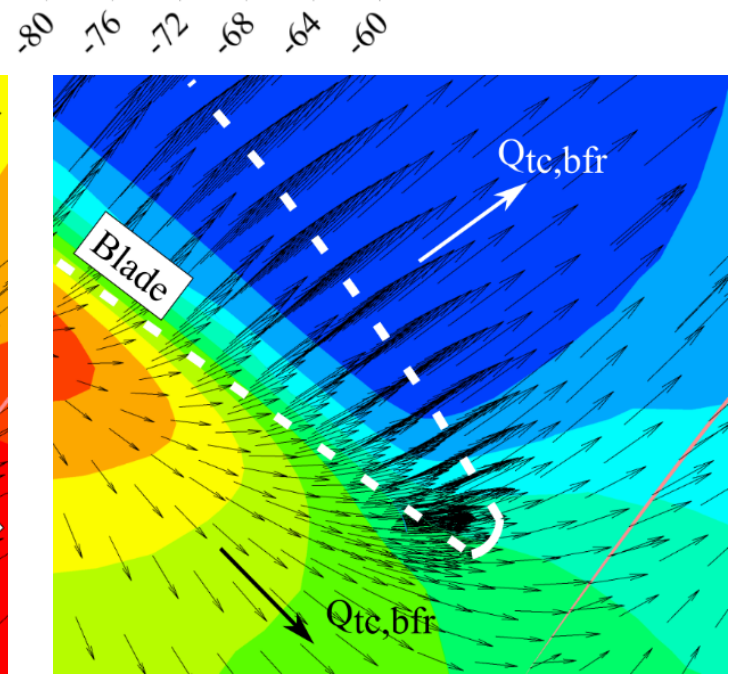

(b) 


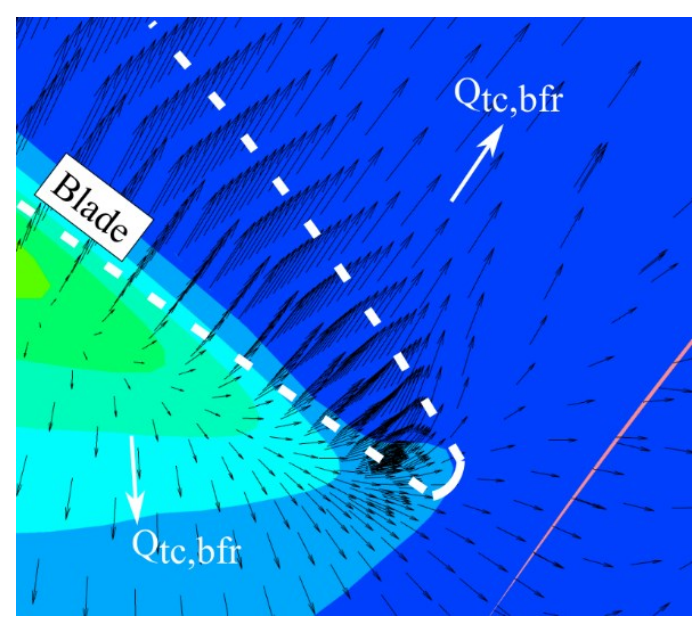

(c)

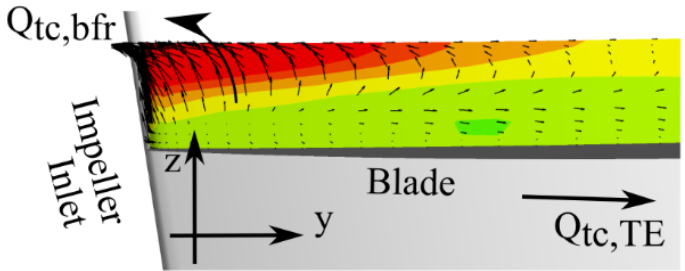

(d)

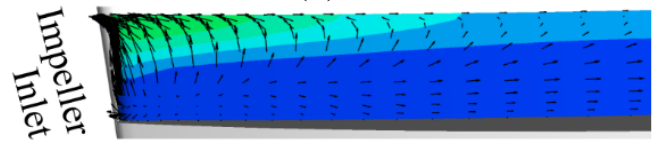

(e)

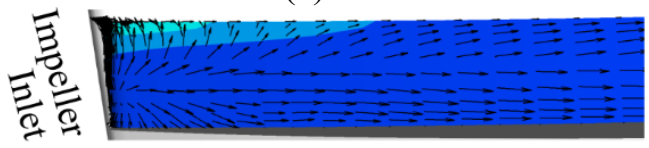

(f)

Fig. 20 Static pressure contour and relative velocity vectors for $t_{t c}=0.9 \mathrm{~mm}$ and $\Phi=0.091$ at (a) $\mathrm{A}_{1}, \sigma_{1}$, (b) $\mathrm{A}_{1}, \sigma_{2}$, (c) $\mathrm{A}_{1}, \sigma_{\mathrm{FC}}$, (d) $\mathrm{A}_{2}, \sigma_{1}$, (e) $\mathrm{A}_{2}, \sigma_{2}$, (f) $\mathrm{A}_{2}, \sigma_{\mathrm{FC}}$.

Moreover, at the $\sigma_{2}$ value, it can be seen that the flow cavitates in the majority of the area between the blade tip and the casing, while the inner and the back flow recirculation, transfers this two-phase flow towards the impeller inlet and suction side and forms backflow cavitation.

However, when $\sigma$ takes its lowest value, and $\Psi$ is collapsed, both recirculation flows exhibit a decrease (Fig. 20c,f). This is because the static pressure continues to decrease only at the pressure side of the blade, and hence the pressure differences which drive backflow recirculation become smaller. As a result, the backflow cavitation weakens as $\sigma$ decreases and hence this flow mechanism seems to affect more the OPs at intermediate values of $\sigma$, around $\sigma_{2}$. This is observed also in [65], where a similar numerical investigation (CFD code and two phase model) is carried out in a turbopump inducer.
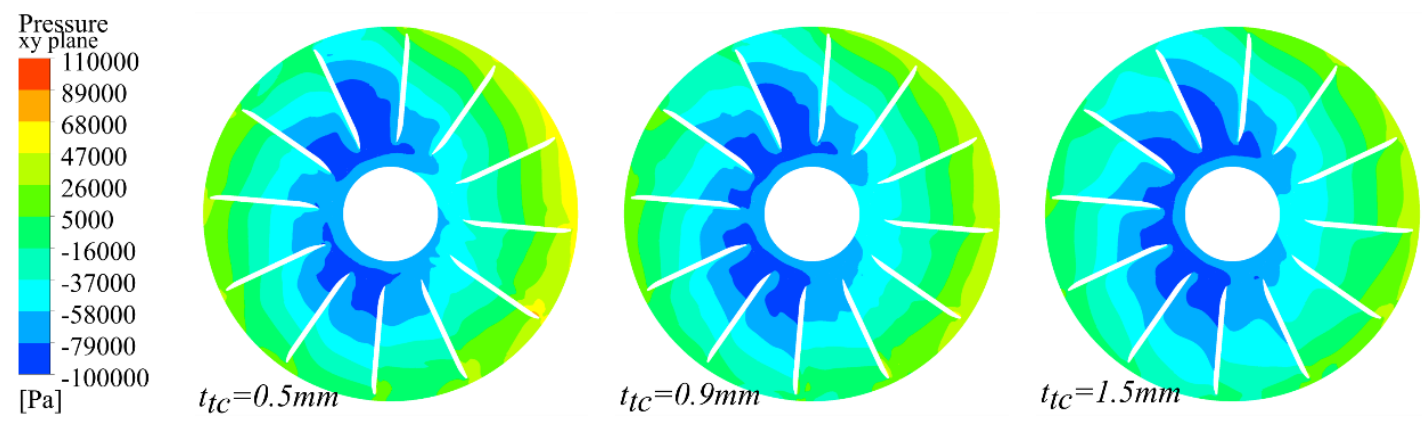

Fig. 21 Pressure contour at $x y$ plane, $z=1 \mathrm{~mm}$ for $\Phi=0.076, \sigma \sim 0.31$ at the three tip clearances.

Finally, the effect of tip clearance thickness on the two phase flow development is investigated with the simulation of two additional geometries of tip clearance under cavitating conditions at $\Phi=0.076$. Three $\sigma$ values are used for the two phase flow analysis that correspond to initial stage $\left(\sigma_{1} \sim 0.42\right)$, intermediate $\left(\sigma_{2} \sim 0.32\right)$ and intense cavitating conditions $\left(\sigma_{F C} \sim 0.13\right)$. In order to achieve identical non-dimensional suction conditions $(\sigma)$ the pressure outlet boundary condition was regulated. The comparison of the static pressure contours at a $x y$ plane for $\sigma_{2} \sim 0.31$, is made in Fig. 21, where a representative view from inside the impeller can be observed $(z=1 \mathrm{~mm})$. The general behavior of the flow is similar for the three tested $t_{t c}$, as the increase of flow recirculation in the larger $t_{t c}$ case is balanced by the flow deceleration due to the larger cross section area.

However, closer examination of the pressure fields in Fig. 21, shows that the extent of lower pressure area in the suction side of the blades increases as $t_{t c}$ is increased. In addition, the relative velocity vectors are plotted in Fig. 22 along with the static pressure contours, in order to depict 
the size of two phase flow area at the tip clearance. According to the results, it can be observed that the low pressure area covers the largest part of the tip clearance at $t_{t c}=0.5 \mathrm{~mm}$, where the cavities extend to the stationary casing side of the numerical domain (Fig. 22a). For larger thicknesses the two-phase cavitating area remains about the same, thus it covers less portion of the tip clearance space $\left(75 \%\right.$ of $t_{t c}=0.9 \mathrm{~mm}$ and $50 \%$ of $\left.t_{t c}=1.5 \mathrm{~mm}\right)$. Also, the flow recirculation area, depicted from the relative velocity vectors in Fig. 22, increases with the increase of $t_{t c}$ similarly to Fig. 15 and strengthens two phase flow formation at impeller's inlet.

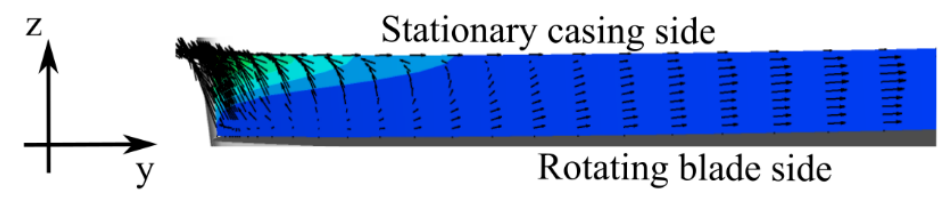

(a)

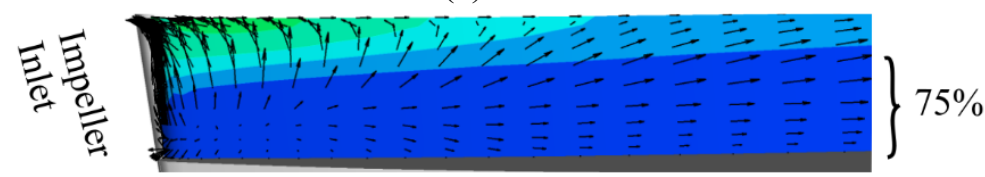

(b)

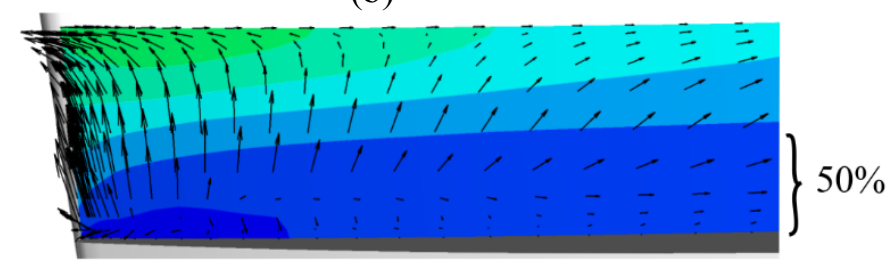

(c)

Fig. 22 Static pressure contour and relative velocity vectors for the three tip clearances modelled, at $\sigma_{2} \sim 0.31$ at $\mathrm{A}_{1}$ for (a) $t_{t c}=0.5 \mathrm{~mm}$, (b) $t_{t c}=0.9 \mathrm{~mm}$, (c) $t_{t c}=1.5 \mathrm{~mm}$.

\section{Conclusions}

In this work a numerical model is developed for the simulation of the performance of a centrifugal pump with radial semi-open impeller, under single and two phase, cavitating flow conditions. For the simulation of the two phase flow the homogeneous approach is implemented with the use of an additional transport equation that is based on the expressions developed from Zwart et al. [36]. The computational domain includes the blades tip clearance area in order to study the effect of tip leakage flows in the performance and suction behavior of the pump. The model is found capable to predict sufficiently the characteristic curves of the pump both under normal operation and cavitating conditions. In addition, flow visualization and laboratory measurements performed for this particular pump confirm the ability of the numerical model to reproduce the total head drop curve due to cavitation, and to predict the location and the extent of the cavitation zone developed for various operating conditions of the pump. The difficulties of the numerical model to reproduce the dynamic part of the vapour area and the differences observed at the total head breakdown operating point, which appear systematically at slightly lower cavitation numbers compared to the experimental data, reflect the limitations introduced in the present methodology by the conduction of steady state simulations and the ignorance of the effect of non-condensable gas in the mixture medium. Nevertheless, the numerical results provide a reliable qualitative view of the complex two-phase flow pattern even at at such severe cavitating conditions.

The flow pattern at the blades tip clearance region includes important secondary flows that recirculate part of the tip flow back to the suction side of the blade and back to the inlet of the impeller. Both these recirculating flows disturb the main flow field in the impeller and create conditions that favour cavitation creation and development. Especially $Q_{t c, b f r}$, recirculates flow 
from the tip cavitating area and brings it towards the cavitated impeller inlet by forming the backflow phenomenon that reinforces the existing two phase flow. The effect of backflow cavitation in the suction performance of the pump is strong until the $\sigma_{F C} \mathrm{OP}$, where the pressure differences that drive recirculation drop, following the total head breakdown. Increase of the tip clearance thickness causes further reduction of the total head and the efficiency of the pump under single phase conditions. Moreover, under cavitating conditions, the tip clearance thickness does not affect considerably the absolute size of the two-phase flow region between the blade tip and the casing, but a larger thickness creates stronger back recirculation flow, which enhances the backflow cavitation behavior of the impeller.

More elaborate numerical modelling, including unsteady simulations and transient effects, presence of non-condensable gases, compressibility effects on turbulence model, etc., would allow for a more detailed analysis and accurate prediction of the complex development and dynamics of cavitation mechanism in centrifugal pumps, from its inception to the head breakdown.

\section{Nomenclature}

\begin{tabular}{ll|ll}
$b_{1}$ & Height blade at the inlet [mm] & $t_{\infty}$ & Mean flow time scale [s] \\
\hline$f_{n c g}$ & Non-condensable gas mass fraction [-] & $u_{1}$ & $\begin{array}{l}\text { Circumferential velocity at the } \\
\text { inlet of the impeller }\left[\mathrm{m} \cdot \mathrm{s}^{-1}\right]\end{array}$ \\
\hline$f_{v}$ & Vapour mass fraction [-] & $w$ & Relative velocity $\left[\mathrm{m} \cdot \mathrm{s}^{-1}\right]$ \\
\hline$Q_{t c, b f r}$ & $\begin{array}{l}\text { Tip clearance backflow recirculation } \\
{\left[\mathrm{m}^{3} \cdot \mathrm{s}^{-1}\right]}\end{array}$ & $\alpha_{n c g}$ & $\begin{array}{l}\text { Non-condensable gas volume } \\
\text { fraction }[-]\end{array}$ \\
\hline$R$ & Impeller radius [m] & $\beta_{1}$ & Blade angle [rad] \\
\hline$R 1$ & Impeller radius inlet [m] & $\beta_{l w}$ & Inlet flow angle [rad] \\
\hline$R 2$ & Impeller radius outlet [m] & $\theta$ & $\begin{array}{l}\text { Angle used for the study of the } \\
\text { impeller angular position }[\mathrm{deg}]\end{array}$
\end{tabular}

\section{Abbreviations}

$\begin{array}{llll}B A & \text { Barotropic Approach } & L E & \text { Leading Edge } \\ B E P & \text { Best Efficiency Point } & M S & \text { Midspan } \\ B T B T & \text { Blade to Blade Topology } & \text { NPSH } & \text { Net Positive Suction Head } \\ C F D & \text { Computational Fluid Dynamics } & O P & \text { Operating Point } \\ F C M & \text { Full Cavitation Model } & T E & \text { Trailing Edge } \\ I C M & \text { Interface Capturing Methods } & T E M & \text { Transport equation-based models } \\ I T M & \text { Interface Tracking Methods } & & \end{array}$

\section{Funding}

This research did not receive any specific grant from funding agencies in the public, commercial, or not-for-profit sectors.

\section{References}

[1] C. E. Brennen, Hydrodynamic of pumps, Cambridge University Press, 2011

[2] G. Mousmoulis, J. Anagnostopoulos, D. Papantonis, A review of experimental detection methods of cavitation in centrifugal pumps and inducers, Int. J. Fluid Mach. Syst. 12 (2019) 71-88. https://doi.org/10.5293/IJFMS.2019.12.1.071. 
[3] Y. Chen, S.D. Heister, A numerical treatment for attached cavitation, J. Fluids Eng. Trans. ASME. 116 (1994) 613-618. https://doi.org/10.1115/1.2910321.

[4] L. Liu, J. Li, Z. Feng, A numerical method for simulation of attached cavitation flows, Int. J. Numer. Methods Fluids. 52 (2006) 639-658. https://doi.org/10.1002/fld.1192.

[5] K. Hejranfar, E. Ezzatneshan, K. Fattah-Hesari, A comparative study of two cavitation modeling strategies for simulation of inviscid cavitating flows, Ocean Eng. 108 (2015) 257275. https://doi.org/10.1016/j.oceaneng.2015.07.016.

[6] M. Deshpande, J. Feng, C.L. Merkle, Cavity flow predictions based on the euler equations, J. Fluids Eng. Trans. ASME. 116 (1994) 36-44. https://doi.org/10.1115/1.2910238.

[7] R. Hirschi, P. Dupont, F. Avellan, J.N. Favre, J.F. Guelich, W. Handloser, E. Parkinson, Centrifugal pump performance drop due to leading edge cavitation: Numerical predictions compared with model tests, Am. Soc. Mech. Eng. Fluids Eng. Div. FED. 11 (1997). https://doi.org/10.1115/1.2820727.

[8] Y. Chen, S.D. Heister, Modeling hydrodynamic nonequilibrium in cavitating flows, J. Fluids Eng. Trans. ASME. 118 (1996) 172-178. https://doi.org/10.1115/1.2817497.

[9] S. Gopalan, J. Katz, Flow structure and modeling issues in the closure region of attached cavitation, Phys. Fluids. 12 (2000) 895-911. https://doi.org/10.1063/1.870344.

[10] Y. Dellanoy, J. L. Kueny, Two phase flow approach in unsteady cavitation modeling. ASME FED (1990), 98, 153-158.

[11] C. Pascarella, V. Salvatore, Numerical study of unsteady cavitation on a hydrofoil section using a barotropic model, Proc. Fourth Int. Symp. Cavitation. (2001) 12.

[12] O. Coutier-Delgosha, B. Stutz, A. Vabre, S. Legoupil, Analysis of cavitating flow structure by experimental and numerical investigations, Journal of Fluid Mechanics. 578 (2007) 171222. https://doi.org/10.1017/S0022112007004934.

[13] O. Coutier-Delgosha, R. Fortes-Patella, J.L. Reboud, Evaluation of the turbulence model influence on the numerical simulations of unsteady cavitation, J. Fluids Eng. Trans. ASME. 125 (2003) 38-45. https://doi.org/10.1115/1.1524584.

[14] S. Frikha, O. Coutier-Delgosha, J.A. Astolfi, Influence of the cavitation model on the simulation of cloud cavitation on 2D foil section, Int. J. Rotating Mach. (2008). https://doi.org/10.1155/2008/146234.

[15] S. Barre, J. Rolland, G. Boitel, E. Goncalves, R.F. Patella, Experiments and modeling of cavitating flows in venturi: attached sheet cavitation, Eur. J. Mech. B/Fluids. 28 (2009) 444 464. https://doi.org/10.1016/j.euromechflu.2008.09.001.

[16] M. Blume, R. Skoda, Numerical 3D analysis of cloud cavitation shedding frequency on a circular leading edge hydrofoil with a barotropic cavitation model, J. Phys. Conf. Ser. 656 (2015). https://doi.org/10.1088/1742-6596/656/1/012146.

[17] U. Jese, R. Fortes-Patella, S. Antheaume, High head pump-turbine: Pumping mode numerical simulations with a cavitation model for off-design conditions, IOP Conf. Ser. Earth Environ. Sci. 22 (2014). https://doi.org/10.1088/1755-1315/22/3/032048.

[18] P. Limbach, T. Müller, R. Skoda, Application of a compressible flow solver and barotropic cavitation model for the evaluation of the suction head in a low specific speed centrifugal pump impeller channel, J. Phys. Conf. Ser. 656 (2015). https://doi.org/10.1088/17426596/656/1/012065.

[19] H.L. Liu, D.X. Liu, Y. Wang, X.F. Wu, J. Wang, Application of modified $\kappa-\omega$ model to predicting cavitating flow in centrifugal pump, Water Science and Engineering. 6(3) (2013) 331-339. https://doi.org/10.3882/j.issn.1674-2370.2013.03.009.

[20] J.B. Leroux, O. Coutier-Delgosha, J.A. Astolfi, A joint experimental and numerical study of mechanisms associated to instability of partial cavitation on two-dimensional hydrofoil, Phys. Fluids. 17 (2005) 1-20. https://doi.org/10.1063/1.1865692.

[21] M. Dular, O. Coutier-Delgosha, Numerical modelling of cavitation erosion, Int. J. Numer. Methods Fluids. 61 (2009) 1388-1410. https://doi.org/10.1002/fld.2003.

[22] D. Zhang, W. Shi, D. Pan, M. Dubuisson, Numerical and experimental investigation of tip leakage vortex cavitation patterns and mechanisms in an axial flow pump, Journal of Fluids Engineering. 137(12) (2015) 121103. https://doi.org/10.1115/1.4030914. 
[23] B. Ji, X. Luo, Y. Wu, X. Peng, Y. Duan, Numerical analysis of unsteady cavitating turbulent flow and shedding horse-shoe vortex structure around a twisted hydrofoil, Int. J. Multiph. Flow. 51 (2013) 33-43. https://doi.org/10.1016/j.ijmultiphaseflow.2012.11.008.

[24] A. Niedźwiedzka, G.H. Schnerr, W. Sobieski, Review of numerical models of cavitating flows with the use of the homogeneous approach, Archives of Thermodynamics. 37(2) (2016) 71-88. https://doi.org/10.1515/aoter-2016-0013.

[25] R.F. Kunz, D.A Boger, D.R Stinebring, T.S Chyczewski, J.W. Lindau, H.J Gibeling, S. Venkateswaran, S., T.R. Govindan, A preconditioned Navier-Stokes method for two-phase flows with application to cavitation prediction, Computers \& Fluids. 29(8) (2000) 849-875. https://doi.org/10.1016/S0045-7930(99)00039-0.

[26] A.K. Singhal, M.M. Athavale, H. Li, Y. Jiang, Mathematical basis and validation of the full cavitation model, Journal of fluids engineering. 124(3) (2002) 617-624. https://doi.org/10.1115/1.1486223.

[27] M. Greenspan, C.E. Tschiegg, Radiation-induced acoustic cavitation; apparatus and some results, J. Res. Natl. Bur. Stand. Sect. C Eng. Instrum. 71C (1967) 299. https://doi.org/10.6028/jres.071c.024.

[28] R. E. Arndt, Cavitation in fluid machinery and hydraulic structures, Annual Review of Fluid Mechanics. 13(1) (1981) 273-326.

[29] J. P. Best, The formation of toroidal bubbles upon the collapse of transient cavities, Journal of Fluid Mechanics. 251 (1993) 79-107. https://doi.org/10.1017/S0022112093003349.

[30] D.J. Duke, M. Battistoni, A.B. Swantek, N. Sovis, A.L. Kastengren, C.F. Powell, S. Som, D.P. Schmidt, Validation of Cavitation Simulations in Submerged Nozzles Energy Systems Division Argonne National Laboratory University of Perugia , Italy X-ray Science Division Argonne National Laboratory Department of Mechanical and Industrial Engineering University o, (n.d.) $1-15$.

[31] J. Jablonska, M. Kozubkova, Methodology of Cavitation Modelling in Diffusers, J. Comput. Multiph. Flows. 7 (2015) 97-109. https://doi.org/10.1260/1757-482X.7.2.97.

[32] S. Yang, C. Habchi, Real-fluid phase transition in cavitation modeling considering dissolved non-condensable gas, Phys. Fluids. 32 (2020). https://doi.org/10.1063/1.5140981.

[33] W. G. Li, Validating full cavitation model with an experimental centrifugal pump. Task Quarterly (2014), 18(1), 81-100.

[34] R.B. Medvitz, R.F. Kunz, D.A. Boger, J.W. Lindau, A.M. Yocum, L.L. Pauley, Performance analysis of cavitating flow in centrifugal pumps using multiphase CFD, Journal of Fluids Engineering. 124(2) (2002) 377-383. https://doi.org/10.1115/1.1457453.

[35] Y. Wang, H. Liu, D. Liu, S. Yuan, J. Wang, L. Jiang, Application of the two-phase threecomponent computational model to predict cavitating flow in a centrifugal pump and its validation, Computers \& Fluids. 131 (2016) 142-150. https://doi.org/10.1016/j.compfluid.2016.03.022.

[36] P.J. Zwart, A.G. Gerber, T. Belamri, A two-phase flow model for predicting cavitation dynamics, In Fifth international conference on multiphase flow. 152 (2004).

[37] G.H. Schnerr, J. Sauer, Physical and numerical modeling of unsteady cavitation dynamics, Fourth International Conference on Multiphase Flow. (2001).

[38] T. Sato, T. Nagahara, S. Suzuki, K. Tanaka, M. Fuchiwaki, M. Nishi, Cavitation analysis on double-suction volute pump, In Proceeding of 3rd IAHR International Meeting of the Workgroup on Cavitation and Dynamic Problems in hydraulic Machinery and Systems. 1 (2009) 185-193.

[39] N.H. Mostafa, M.A. Boraey, Numerical and experimental investigation of cavitation in axial pumps, In 2006 8th International Congress of Fluid Dynamics and Propulsion. (2006) 14-17.

[40] H. Ding, F.C. Visser, Y. Jiang, M. Furmanczyk, Demonstration and validation of a 3D CFD simulation tool predicting pump performance and cavitation for industrial applications, Journal of fluids engineering. 133(1) (2011) 277-293. https://doi.org/10.1115/FEDSM2009-78256.

[41] J.S. Anagnostopoulos, A fast numerical method for flow analysis and blade design in centrifugal pump impellers, Computers \& Fluids. 38(2) (2009) 284-289. 
https://doi.org/10.1016/j.compfluid.2008.02.010.

[42] M.J. Kim, H.B. Jin, W.J. Chung, A study on prediction of cavitation for centrifugal pump, World Acad. Sci. 6(12) (2012) 612-617.

[43] U. Iben, Modeling of cavitation, Systems Analysis Modelling Simulation. 42(9) (2002) 1283-1307. https://doi.org/10.1080/716067218.

[44] D. Pierrat, L. Gros, G. Pintrand, B. Le Fur, P. Gyomlai, Experimental and numerical investigations of leading edge cavitation in a helico-centrifugal pump, In The 12th International Symposium of Transport Phenomena and Dynamics on Rotating Machinery. (2008) 17-22.

[45] J. Liu, S. Liu, Y. Wu, L. Jiao, L. Wang, Y. Sun, Numerical investigation of the hump characteristic of a pump-turbine based on an improved cavitation model, Computers \& Fluids. 68 (2012) 105-111. https://doi.org/10.1016/j.compfluid.2012.08.001.

[46] M.A.R. Cunha, H.F.V. Nova, Cavitation modelling of a centrifugal pump impeller, In 22nd International Congress of Mechanical Engineering. (2013).

[47] D. Zhang, W. Shi, B.B. van Esch, L. Shi, M. Dubuisson, Numerical and experimental investigation of tip leakage vortex trajectory and dynamics in an axial flow pump, Computers \& fluids. 112 (2015) 61-71. https://doi.org/10.1016/j.compfluid.2015.01.010.

[48] D. Zhang, L. Shi, W. Shi, R. Zhao, H. Wang, B.B. van Esch, Numerical analysis of unsteady tip leakage vortex cavitation cloud and unstable suction-side-perpendicular cavitating vortices in an axial flow pump, International Journal of Multiphase Flow. 77 (2015) 244-259. https://doi.org/10.1016/j.ijmultiphaseflow.2015.09.006.

[49] G. Mousmoulis, I. Kassanos, J. Anagnostopoulos, D. Papantonis, Experimental and numerical investigation of the cavitating draft tube vortex in a Francis model turbine, 6th IAHR International Meeting of the Workgroup on Cavitation and Dynamic Problems in Hydraulic Machinery and Systems. (2015).

[50] I. Kassanos, J. Anagnostopoulos, D. Papantonis, Numerical analysis of the effect of splitter blades on draft tube cavitation of a low specific speed Francis turbine, 6th IAHR International Meeting of the Workgroup on Cavitation and Dynamic Problems in Hydraulic Machinery and Systems. (2015).

[51] K.J. Farrell, M.L. Billet, A correlation of leakage vortex cavitation in axial-flow pumps, Journal of fluids engineering. 116(3) (1994) 551-557. https://doi.org/10.1115/1.2910312.

[52] R. Laborde, P. Chantrel, M. Mory, Tip clearance and tip vortex cavitation in an axial flow pump, Journal of fluids engineering. 119(3) (1997) 680-685. https://doi.org/10.1115/1.2819298.

[53] C. Yang, C. Chen, J. Wang, Q. Ji, Three-dimensional viscous numerical simulation of tip clearance flow in axial-flow pump, Journal of Thermal Science. 12(3) (2003). https://doi.org/10.1007/s11630-003-0073-1.

[54] S.Q. Wu, W.D. Shi, D.S. Zhang, J. Yao, C. Cheng, Influence of blade tip rounding on tip leakage vortex cavitation of axial flow pump, In IOP Conference Series: Materials Science and Engineering. 52(6) (2013). https://doi.org/10.1088/1757-899X/52/6/062011.

[55] J. Feng, X. Luo, P. Guo, G. Wu, Influence of tip clearance on pressure fluctuations in an axial flow pump, Journal of Mechanical Science and Technology. 30(4) (2016) 1603-1610.

[56] Y. Xu, L. Tan, Y. Liu, S. Cao, Pressure fluctuation and flow pattern of a mixed-flow pump with different blade tip clearances under cavitation condition, Advances in Mechanical Engineering. 9(4) (2017). https://doi.org/10.1177/1687814017696227.

[57] Y. Hao, L. Tan, Y. Liu, Y. Xu, J. Zhang, B. Zhu, Energy performance and radial force of a mixed-flow pump with symmetrical and unsymmetrical tip clearances, Energies. 10(1) (2017) 57. https://doi.org/10.1007/s12206-016-0315-2.

[58] Y. Liu, L. Tan, Y. Hao, Y. Xu, Energy performance and flow patterns of a mixed-flow pump with different tip clearance sizes, Energies. 10(2) (2017) 191. https://doi.org/10.3390/en10020191.

[59] T. Lei, X. Zhifeng, L. Yabin, H. Yue, X. Yun,Influence of T-shape tip clearance on performance of a mixed-flow pump, Proceedings of the Institution of Mechanical Engineers, Part A: Journal of power and energy. 232(4) (2018) 386-396. 
[60] W. Zhang, Z. Yu, B. Zhu, Influence of tip clearance on pressure fluctuation in low specific speed mixed-flow pump passage, Energies. 10(2) (2017) 148. https://doi.org/10.3390/en10020148.

[61] Y. Hao, L. Tan, Symmetrical and unsymmetrical tip clearances on cavitation performance and radial force of a mixed flow pump as turbine at pump mode, Renewable energy. 127 (2018) 368-376. https://doi.org/10.1016/j.renene.2018.04.072.

[62] Y. Liu, L. Tan, Tip clearance on pressure fluctuation intensity and vortex characteristic of a mixed flow pump as turbine at pump mode, Renewable energy. 129 (2018) 606-615. https://doi.org/10.1016/j.renene.2018.06.032.

[63] T. Hashimoto, M. Yoshida, M. Watanabe, Experimental study on rotating cavitation of rocket propellant pump inducers, Journal of Propulsion and Power. 13(4) (1997) 488-494. https://doi.org/10.2514/2.5210.

[64] S.S. Hong, J.S. Kim, C.H. Choi, J. Kim, Effect of tip clearance on the cavitation performance of a turbopump inducer, Journal of Propulsion and Power. 22(1) (2006) 174179. https://doi.org/10.2514/1.11982.

[65] S. Kim, C. Choi, J. Kim, J. Park, J. Baek, Tip clearance effects on cavitation evolution and head breakdown in turbopump inducer, Journal of Propulsion and Power. 29(6) (2013) 1357 1366. https://doi.org/10.2514/1.B34766.

[66] F. Bakir, R. Rey, A. Gerber, T. Belamri, B. Hutchinson, Numerical and Experimental Investigations of the Cavitating Behavior of an Inducer, Int. J. Rotating Mach. 10 (2004) 15-25. https://doi.org/10.1080/10236210490258034.

[67] P. Limbach, R. Skoda, Numerical and Experimental Analysis of Cavitating Flow in a Low Specific Speed Centrifugal Pump with Different Surface Roughness, J. Fluids Eng. Trans. ASME. 139 (2017). https://doi.org/10.1115/1.4036673.

[68] F.R. Menter, Two-equation eddy-viscosity turbulence models for engineering applications, AIAA journal. 32(8) (1994) 1598-1605. https://doi.org/10.2514/3.12149.

[69] International Organization for Standardization 2012, Rotodynamic pumps: Hydraulic performance acceptance tests: Grades 1, 2 and 3, ISO 9906:2012, International Organization for Standardization, Geneva.

[70] F. Zhang, S. Yuan, Q. Fu, J. Pei, M. Böhle, X. Jiang, Cavitation-induced unsteady flow characteristics in the first stage of a centrifugal charging pump, Journal of Fluids Engineering. 139(1) (2017) 011303. https://doi.org/10.1115/1.4034362.

[71] G. Mousmoulis, N. Karlsen-Davies, G. Aggidis, I. Anagnostopoulos, D. Papantonis, Experimental analysis of cavitation in a centrifugal pump using acoustic emission, vibration measurements and flow visualization, European Journal of Mechanics-B/Fluids. 75 (2019) 300-311. https://doi.org/10.1016/j.euromechflu.2018.10.015.

[72] G. Mousmoulis, N. Karlsen-Davies, G. Aggidis, J. Anagnostopoulos, D. Papantonis, Experimental analysis of the onset and development of cavitation in a centrifugal pump, In Journal of Physics: Conference Series. 813(1) (2017).

[73] B. Zhu, H. Chen, Analysis of the staggered and fixed cavitation phenomenon observed in centrifugal pumps employing a gap drainage impeller, Journal of Fluids Engineering. 139(3) (2017). https://doi.org/10.1115/1.4034952.

[74] M. Kaya, E. Ayder, Prediction of Cavitation Performance of Radial Flow Pumps, Journal of Applied Fluid Mechanics. 10(5) (2017). https://doi.org/10.18869/acadpub.jafm.73.242.27827.

[75] C. Kim, S. Kim, C.H. Choi, J. Baek, Effects of inducer tip clearance on the performance and flow characteristics of a pump in a turbopump, Proceedings of the Institution of Mechanical Engineers, Part A: Journal of Power and Energy. 231(5) (2017) 398-414.

[76] G.M. Wood, H. Welna, R.P. Lamers, Tip-clearance effects in centrifugal pumps, Journal of basic engineering. 87(4) (1965) 932-939. https://doi.org/10.1115/1.3650846.

[77] G. Boitel, D. Fedala, N. Myon, Tip clearance effects on loads and performances of semiopen impeller centrifugal pumps at different specific speeds, In IOP Conference Series: Earth and Environmental Science. 49(3) (2016).

https://doi.org/10.1088/1755-1315/49/3/032013.

[78] J.F. Gülich Centrifugal pumps, Springer, Berlin, 2008. 
[79] J.W. Lindau, W.L. Moody, M.P. Kinzel, J.J. Dreyer, R.F. Kunz, E.G. Paterson, Computation of cavitating flow through marine propulsors, In Proceedings of the 1st International Symposium on Marine Propulsors. (2009).

[80] J. Wack, S. Riedelbauch, Cavitation Simulations of a Tip Leakage Vortex for a NACA0009 Hydrofoil and a Francis Turbine at Stable Full Load Operating Point, In High Performance Computing in Science and Engineering'18. (2019) 351-365. https://doi.org/10.1007/978-3-030-13325-2_22.

[81] D.Q. Li, Prediction of non-cavitating and cavitating performance of a SVA Potsdam propeller, In Second International Symposium on Marine Propulsors. Hamburg University of Technology Hamburg. (2011).

[82] Z.J. Jin, Z.X. Gao, X.J. Li, J.Y. Qian, Cavitating Flow through a Micro-Orifice, Micromachines. 10(3) (2019) 191.

[83] C. E. Brennen, Cavitation and bubble dynamics, Cambridge University Press, 2014. 